\title{
Mechanics and Energetics Modeling of Ball-Milled Metal Foil and Particle Structures
}

Matteo Aureli, Department of Mechanical Engineering, University of Nevada Reno, Reno, Nevada (maureli@unr.edu )

Constantine C. Doumanidis, Pankyprion Gymnasion, Nicosia, Cyprus (kdoumani98@gmail.com)

I.E. Gunduz, School of Mechanical Engineering, Purdue University, West Lafayette, Indiana (igunduz@purdue.edu)

Aseel Gamal Suliman Hussien, Department of Mechanical Engineering, Khalifa University, Abu Dhabi, UAE (aseel.hussien@kustar.ac.ae)

Yiliang Liao, Department of Mechanical Engineering, University of Nevada Reno, Reno, Nevada (yliao@unr.edu)

Claus Rebholz, Department of Mechanical \& Manufacturing Engineering, University of Cyprus, Nicosia, Cyprus (claus@ucy.ac.cy)

Charalabos C. Doumanidis, Department of Mechanical Engineering, Khalifa University, Abu Dhabi, UAE (haris.doumanidis@kustar.ac.ae)

\begin{abstract}
$\underline{\text { Abstract }}$
The reported research establishes a semi-analytical computational predictive model of fractal microstructure in ball-milled metal foils and powder particulates, with emphasis on its transformation mechanics via an energy-based approach. The evolving structure is composed of reconfigurable warped ellipsoid material domains, subjected to collisions with the ball milling impactors following Brownian motion energetics. In the first step of the model, impacts are assumed to generate ideal Hertzian elastic stress fields, with associated bulk deformations quantified as per Castigliano's strain energy methods. In the second stage of the model, elastic energies are recast to produce frictional slip and plastic yield, thus resulting in surface micro-joints. Only two parameters of the model necessitate experimental calibration, performed by comparison of joint energy with laboratory tensile measurements on ballmilled multilayer Al-Ni foils. Model predictions of evolving internal microstructure are validated against SEM micrographs of Al-Ni powder particulate samples for different ball milling durations. Results demonstrate the capability of the model to accurately capture relevant fractal measures of the microstructure of ball-milled powders.
\end{abstract}

\section{Keywords}

Mechanical alloying, ball milling, multilayer foil, particulate, fractal structure.

\section{Introduction and Prior Work}

Over the past three decades the materials community has made groundbreaking progress in mechanochemistry research addressing mechanical alloying (MA) of metals and non-metallic materials. On the one hand, this has enabled a variety of transformative and impactful applications, as e.g. in ball milling (BM) fabrication of bimetallic and multi-material micro- and nano-structures, such as of nickel, iron and titanium aluminides etc., used for self-propagating high-temperature synthesis [1]. Such reactive 
metallic multilayers, upon thermal or electrical ignition, release heat, thus generating adiabatic temperatures of interest to metal joining, soldering and packaging applications [2]. More recently, planetary ball milling of $\mathrm{Ni}$ and $\mathrm{Al}$ powders in both high-energy (thermally transient) [3] and low-energy (thermally steady state) [4] implementation was experimentally researched. This showed promise for scalable manufacturing of self-similar (fractal) particles, ignitable in loose, cold-pressed pellet and sandwiched foil forms [5].

On the other hand, aside from its applications, influential intellectual motivation in MA originated from pioneering work performed through the 90's in establishing a conceptual framework and in numerical modeling of milling tool dynamics and micro-structural evolution of BM particulates [6-9]. These investigations in planetary and vibratory milling elucidated fundamental aspects of BM kinematics and mechanochemistry of the alloyed materials in actual MA devices [7-10]. This seminal research inspired implementation of its basic principles, mathematical and experimental analysis in the context of planetary BM [11,12], horizontal rod mills [13] and BM energy transfer [14,15], eliciting considerable laboratory evidence and further theoretical refinement. Further effort tackled the kinetics of initial stages of MA [16,17], and recently structural refinement modeling $[18,19]$ predicting evolution of lamellar thickness and grain size. Computational research addressed modeling of constitutive material behavior during impact for deformation analysis by finite element methods, as well as simulation of the ball dynamics, using the discrete element method [20]. Revived interest in MA culminated with recent comprehensive reviews [21], enabling new material design, analysis and optimization [2,5].

Computational modeling of MA has been facing the challenges of multi-domain, multi-scale, stochastic chaotic nature of the BM process. Despite valuable insights, however, numerical complexity of such an off-line simulation approach left an outstanding need for novel BM modeling tools with in-process computational efficiency, empowering real-time predictions of the spatially-distributed, time-evolving particulate structure. Such simulation tools would be advantageous as in-process MA observers, i.e. models running in parallel to the BM hardware and providing estimates of mechanically alloyed material states, such as particulate size and shape, structure and composition, and thermal and diffusive condition. Since these are usually inaccessible non-invasively and/or non-destructively in the enclosed, rotating BM setting, real-time model predictions could provide substitute feedback for closed-loop process control schemes. In addition, and beyond lamellar thickness, the full complex topology of material interfaces in the multi-scale, self-similar particulate structure needs to be captured by such modeling. This is because the fractal architecture directly determines the transport properties of MA products, and therefore their operational performance, such as in the case of ignitable reactive bimetallic systems $[3,4]$.

A preliminary framework was recently proposed [22] towards such a real-time, experimentally validated computational simulation of the stochastic dynamics of the full fractal structure distribution in particulates during a BM process. Instead of numerically demanding discrete element methods for explicit BM kinematics, this approach adopts an analytical phenomenological probabilistic formulation of Brownian-like kinetics and energetics of impactor motion [23], matching computational predictions and laboratory measurements in the literature [24]. Analogous new stochastic descriptions of MA surface impact, contact, friction and volume deformation, coalescence and fragmentation mechanisms on universal geometric primitive elements of BM particle domains are also suggested. Instead of redundant vectorial and tensorial mechanics, material constitutive models are based on efficient scalar energy-based stress-strain transformations between ideal reversible and actual irreversible 
configurations. These are intended to preserve the fundamental underlying physics and chemistry of the chaotic BM process, and the concomitant fractal complexity of resulting particulate microstructures.

Thus, the objective of this article is to introduce and implement such novel primitive domain geometries and their energy-based transformations during the chaotic BM process dynamics, towards a real-time predictive simulation of the full fractal structure of MA particulates. The model description framework of a representative particulate composed of warped ellipsoid material domains (section 2.1) introduces a kinetic theory-based formulation of milling ball motion (section 2.2) for simulation of random collisions of the impactors (milling balls and vial walls). The model then departs with initially ideal elastic contact mechanics and energetics of the impact and joint locations of this particulate (section 2.3). These are subsequently recast to predict surface slip and dry friction (section 2.4), as well as plastic yield and strain-hardening of the materials (section 2.5), along with bulk deformation of the domains (section 2.6), generating heat and potentially micro-welded joints. Laboratory calibration of friction and yield parameters is performed experimentally by MA Al-Ni foils subjected to tensile lap testing (section 3.1). The composite model is validated by comparison of predicted microstructures of BM powders with experimental micrographs (section 3.2), with conclusions and suggestions for further work in section 4.

\section{Model Development}

\section{$\underline{2.1 \text { Model Framework and Assumptions }}$}

Morphological observation of ball-milled bimetallic particulate sections reveals a multi-scale self-similar microstructure (Fig. 1a) composed of lamellar network (branching tree) formations, which have evolved by deformation of globular agglomerates (Apollonian packs) of the original metal powders (Fig. 1b). This fractal microstructure transformation during the BM process can be described by the deformation and joining of these individual monometallic particle domains as warped ellipsoid (WE) primitives (Fig. 2). For direct comparison with experimental planar micrographs (e.g. Fig. 1a), the model only focuses on a 2D section of the particulate, whereby each WE is described, to a second order approximation, by only 8 scalar parameters: centroid coordinates $X, Y$; orientation angles $\phi_{x} \phi_{y}$; semi-axes $I_{x} I_{y}$; and curvature radii $R_{x} R_{y}$ (Fig. 2). This $2 \mathrm{D}$ geometric configuration results in computational efficiency of the model over a 3D formulation, which would require 21 parameters for each WE. At the same time, parametric selfsimilarity of the WEs is preserved as they reshape from spheroidal $\left(I_{x} \approx I_{y}, R_{x} \approx R_{y}\right)$ to lamellar $\left(I_{x}>>I_{y}\right.$, $R_{x}>>R_{y}$ ) form, resulting from interaction with impactors, i.e. the BM balls and vial walls, and other particles. The model actually carries out all computation below on extrapolated 3D domains, with semiaxis $l_{z}=\sqrt{l_{x} l_{y}}$ so that equal WE section areas correspond to equal domain volumes, and radius $R_{z}=2 R_{x} R_{y} /\left(R_{x}+R_{y}\right)$ leaving the equivalent local curvature of the section boundary invariant. Initial distributions of WE parameters are interpolated from contours of the initial ball-milled loose powders on actual micrographs.

During BM, the simulation tracks dynamic evolution over time of one representative particulate as it is progressively transformed by deformation of its WE domains, and as its joins with adjacent particles (WEs) and clusters (WE assemblies) over its boundary (Fig. 3). For this purpose, the model adopts a Lagrangian approach, following a moving Cartesian frame ( $x, y$ coordinate system) fixed with respect to the studied particulate. As in Fig. 3, the particulate is surrounded by an adjacent layer of randomly selected particles and clusters, and the assembly is impacted at a random location by BM balls and vial wall impactors, moving at incidence velocity $v$ and orientation $\phi$. The model ignores presence of the 
inert gas atmosphere in the vial, potential liquid process control agents, as well as gravitational and dynamic inertial effects. It also assumes steady-state thermal equilibrium in the vial and its contents (as e.g. in low-energy BM). In other words, isothermal process conditions are considered, neglecting the dependence of domain dimensions and material properties on thermal effects. In addition, material properties are evaluated for an average deformation rate of impact during BM, and strain rate effects are neglected. Absence of material transformations via diffusive and reactive phenomena at interfaces and bulk volumes during the BM process is also presumed.

All particulate materials are assumed homogeneous and isotropic with linear elasto-plastic behavior. In the material bulk, the constitutive behavior is described by a bilinear strain-hardening stress-strain $(\sigma, \varepsilon)$ or ( $\tau, \gamma)$ curve, at elastic moduli $E, G\left(G=E / 2(1+v)\right.$, with $v$ the Poisson ratio), and $E^{\prime}, G^{\prime}$ beyond yield stress $\sigma_{y}, \tau_{y}$ (Fig. $4 a$, thick solid line), resulting in dissipation of volumetric plastic work $W_{p}$ (along dot-dashed unloading line). At contact surfaces, the normal pressure-displacement $(p, \beta)$ curve is similar (Fig. 4a). The shear stress-displacement $(q, \delta)$ saturates at a local level of $\mu . p$ (Fig. $4 b)$, with $\mu$ the Coulomb dry friction coefficient between rubbing materials, resulting in dissipation of surface frictional work $W_{f}$ (along dot-dashed line). Impactor materials, such as stainless steel, ideally deform within their elastic region only.

The model determines all displacements in two distinct stages. Initially, the particulate conceptually absorbs the impacting energy $U_{\text {in }}$ as ideal elastic volumetric or surface work at moduli $E, G$ (area OBI, Fig. $4)$, thus resulting in ideal stresses-strains $\left(\sigma_{i}, \varepsilon_{i}\right),\left(\tau_{i}, \gamma_{i}\right)$. Subsequently, the particulate exhibits plastic deformation and/or surface friction separately and adiabatically, i.e. preserving the total work performed $W_{i}=U_{\text {in }}$ (area $\mathrm{OADH}$, Fig. 4). By recasting such stored energy so that area $\mathrm{OBI}$ equals area $\mathrm{OADH}$, this step relaxes stresses and extends displacements to actual $(\sigma, \varepsilon),(\tau, \gamma)$ at full loading (point $\mathrm{D}$, Fig. 4). During the unloading phase of impact, the model also adopts an ideal elastic behavior at moduli $E, G$, by superposing opposite elastic stresses $\left(-\sigma+\sigma_{r},-\tau+\tau_{r}\right)$ to the full load stresses $(\sigma, \tau)$ along dotdashed line DF in Fig. 4. During such unloading of the contact points, local load equilibrium and displacement compatibility prevent the eventual residual stress and strain fields $\left(\sigma_{r}, \varepsilon_{r}\right),\left(\tau_{r}, \gamma_{r}\right)$ from fully relaxing to $\left(0, \varepsilon_{p}\right),\left(0, \gamma_{f}\right)$. Therefore, only a fraction of the unloading elastic work $W_{e}($ area FDH, Fig. 4$)$ is returned as kinetic energy $U_{\text {out }}=W_{e}-W_{r}$ to the particulate surroundings (area GJDH), as the residual work $W_{r}$ (area FJG) is stored elastically in the material. This residual elastic energy may be subsequently depleted by loading or annealing upon further collision events. During a full impact cycle, an energy balance yields $U_{\text {in }}=W_{i}=W_{p}+W_{f}+W_{e}=W_{p}+W_{f}+W_{r}+U_{\text {out }}=H+W_{r}+U_{\text {out }}$ since $W_{p}+W_{f}$ is dissipated as heat $H$.

\section{$\underline{2.2 \text { Impact Energetics }}$}

To generate the random impactor energies $U_{\text {in }}$ (Figs. 3, 4), the model adopts Brownian-like kinetics of BM motion [23], through a laboratory-calibrated and validated Maxwell-Boltzmann probability density function (pdf) for the impact velocity $v=v_{\text {in }}$ (Figs. 3,5 ):

$$
\Phi(v)=\frac{2 \sqrt{2}}{\sqrt{\pi} s}\left(\frac{v^{2}}{2 s^{2}}\right) \exp \left(-\frac{v^{2}}{2 s^{2}}\right)
$$

where the pdf parameter $s$ is determined from the most probable velocity $v_{p}=\sqrt{2} s$ or the root mean square (rms) average $\sqrt{\left\langle v^{2}\right\rangle}=\sqrt{3} s$ of the experimentally measured velocity distribution (Fig. 5). The equivalent two-body system collision mass $m^{*}$ of an impactor (BM ball or vial wall) of actual mass $m^{\prime}$ colliding with a particulate of mass $m$ can be determined as in [25], where typically $m^{\prime}>>m$ : 
Therefore, for randomly chosen impact velocity $v_{\text {in }}$ (selected from Eq. 1 ) and orientation angle $\phi$ (selected from a uniform pdf over the incidence range $0 . . . \pi$ ), the corresponding energy $U_{i n}$ is determined as:

$$
U_{\text {in }}=\frac{1}{2} m^{*} v_{\text {in }}^{2}=\frac{1}{2} m^{*} v_{n}^{2}+\frac{1}{2} m^{*} v_{t}^{2}=U_{n}+U_{t} \quad \text { (Eq. 3) }
$$

where $v_{n}, v_{t}$ and $U_{n}, U_{t}$ are the normal and tangential components of impact velocity and energy, respectively, i.e. (Fig. 3)

$$
v_{n}=v_{i n} \sin \phi, v_{t}=v_{\text {in }} \cos \phi \quad \text { and } \quad v_{\text {in }}^{2}=v_{n}^{2}+v_{t}^{2} \quad \text { (Eq. 4) }
$$

\subsection{Contact Surface Elastic Fields}

In its initial ideal elastic step, the model adopts a modified Hertzian [26-30] approach in determining the elastic field energy during the loading and unloading phases of impactor-particulate and particulateparticulate (i.e. particle or cluster) contacts. In both cases, the effective contact curvature radius $R *$ is computed from the equivalent radii $R_{1}$ and $R_{2}$ of the contacting sides as:

$$
R_{*}=\frac{R_{1} R_{2}}{R_{1}+R_{2}} \text { where } R_{1,2}=2\left(\frac{R_{a 1,2} R_{b 1,2}}{R_{a 1,2}+R_{b 1,2}}\right)
$$

where $R_{a}, R_{b}$ are the principal radii of curvature of each side $(1,2)$ at the contact point (e.g. $R_{\text {ball }}=R$ for a ball impactor (Fig. 3), $R_{c y l}=2 R_{\text {vial }}$ for the cylindrical vial wall and $R_{c a p}=\infty$ for flat cover walls). The effective normal and shear moduli of elastic contact $E *$ and $G *$ are also determined as:

$$
E_{*}=\frac{E_{1} E_{2}}{E_{1}\left(1-v_{2}^{2}\right)+E_{2}\left(1-v_{1}^{2}\right)}, G_{*}=\frac{G_{1} G_{2}}{G_{1}\left(1-v_{2}^{2}\right)+G_{2}\left(1-v_{1}^{2}\right)} \quad \text { (Eq. 6) }
$$

from the elastic moduli $E_{1,2}, G_{1,2}$ and Poisson ratios $v_{1,2}$ of the two sides $(1,2)$. Beyond the yielding point (Fig. 4a), the elastic moduli above are replaced with the strain-hardening moduli $E_{1,2}^{\prime} G_{1,2}^{\prime}$ for computation of the additional elastic fields.

For a general elastic (no yield or slip) two-side contact at normal load $P$ and shear load $Q$ (determined below for each contact type), Hertzian contact dictates that the contact surfaces deform into disks of radius $\alpha$ and $a$ respectively, determined as (Fig. 6):

$$
\alpha=\sqrt[3]{\frac{3 P R_{*}}{4 E_{*}}} \text { and } a=\min \left[\sqrt[3]{\frac{3 Q R_{*}}{4 G_{*}}}, \alpha\right]
$$

Over the plane of these disks, Hertzian contact prescribes a semi-elliptical distribution of normal pressure $p(r)$ and shear stress $q(r)$, with maximum stress values $p_{o}=\frac{3}{2} \frac{P}{\pi \alpha^{2}}$ and $q_{o}=\frac{3}{2} \frac{Q}{\pi a^{2}}$ respectively, of the form:

$$
p(r)=p_{o} \sqrt{1-\left(\frac{r}{\alpha}\right)^{2}}
$$


where $r$ denotes the distance in the plane of the disk from the contact axis. Normal elastic displacement $\beta(r)$ and shear elastic displacement $\delta(r)$ of the contact surface are given respectively by:

$$
\begin{aligned}
& \beta(r)=\frac{2 \alpha^{2}-r^{2}}{2 R_{*}} \quad \text { (Eq. 10) } \\
& \delta(r)=\frac{2 a^{2}-r^{2}}{2 R_{*}} \quad(\text { Eq. 11) }
\end{aligned}
$$

with maximum normal and shear displacements given by $\beta_{o}=\frac{\alpha^{2}}{R_{*}}$ and $\delta_{o}=\frac{a^{2}}{R_{*}}$, respectively.

The contact stiffness in the normal $K_{n}$ and shear $K_{t}$ direction can thus be computed as:

$$
K_{n}=\frac{\partial P}{\partial \beta_{o}}=2 E_{*} \alpha, K_{t}=\frac{\partial Q}{\partial \delta_{o}}=2 G_{*} a \rightarrow P \approx K_{n} \beta_{o}, Q \approx K_{t} \delta_{o} \quad \text { (Eq. 12) }
$$

For an impactor-particulate contact, the typically much larger size of the impactor (BM ball or vial wall) causes absorption of the impact energy $U_{\text {in }}$ by the entire volume of the smaller particulate and its surrounding layer of particles and clusters ("surrounding medium", Fig. 3). Thus, the model assumes that the normal pressure $P$ and shear load $Q$ at the elastic contact are developed via absorption of the impact energy components $U_{n}, U_{t}$ by these WE constituents according to a compliance-weighted volumetric rule:

$$
P=\pi \alpha^{2} \sqrt{2 U_{n}\left(\frac{E}{V}\right)_{*}} \quad \text { and } \quad Q=\pi a^{2} \sqrt{2 U_{t}\left(\frac{G}{V}\right)_{*}} \quad \text { (Eq. 13) }
$$

where the effective volumetric stiffness values $(E / V) *$ and $(G / V) *$ are calculated as:

$$
\left(\frac{E}{V}\right)_{*}=\left(\sum_{i=1}^{n} \frac{V_{i}}{E_{i}}\right)^{-1} \text { and }\left(\frac{G}{V}\right)_{*}=\left(\sum_{i=1}^{n} \frac{V_{i}}{G_{i}}\right)^{-1} \text { (Eq. 14) }
$$

for the volumes $V_{i}$ and elastic moduli $E_{i}$ and $G_{i}$ of all the $n$ WE constituents, $i=1 \ldots n$ in the particulate and its surrounding medium. Therefore, for the impactor-particulate contact the previous equations yield:

$$
\alpha=\frac{3 \pi R_{*}}{4 E_{*}} \sqrt{2 U_{n}\left(\frac{E}{V}\right)_{*}} \text { and } a=\min \left[\frac{3 \pi R_{*}}{4 G_{*}} \sqrt{2 U_{t}\left(\frac{G}{V}\right)_{*}}, \alpha\right]
$$

For particulate-particulate contacts, instead of considering the energetics of the elastic impact (Eq. 13), the loads $P, Q$ are computed via the stiffness relationships (Eq. 12), from the elastic displacements $\beta, \delta$ determined via the bulk deformation analysis described below. In both cases, the effective total elastic field volume is $V=E * /(E / V) *$ (see Eqs. 6 and 14$)$.

\section{$\underline{2.4 \text { Contact Surface Slip and Friction }}$}

After the ideal elastic step is completed for the loading phase of each impact, in its subsequent inelastic steps, the model detects slip conditions over the contact surfaces. General slip in dry friction occurs as $\alpha=a$ along with $Q / P>\mu$. Therefore, Eq. 7 shows that, as the $Q / P$ ratio increases, the general slip condition is given by: 


$$
\frac{Q}{P} \geq \frac{G_{*}}{E_{*}}=\frac{1}{2\left(1+v_{*}\right)} \quad \text { i. e. } \quad \frac{Q}{P} \geq \max \left[\mu, \frac{1}{2\left(1+v_{*}\right)}\right] \equiv M
$$

where $v_{*}=\left(E_{*} / 2 G_{*}\right)-1$. In particular, when $Q / P \geq 1 / 2\left(1+\nu_{*}\right)$, the disk of shear stress distribution extends to cover the entire disk of normal stress distribution (Eq. 7), and $a$ saturates at $a=\alpha$, creating one necessary condition for general slip of the full contact surface. Thus, when in addition $q_{o} / p_{o}=Q / P \geq M$, the second necessary condition is met, and full slip occurs over the entire contact disk. However, local micro-slip initiates at the contact center as soon as $q_{o}>\mu p_{o}$ (Fig. 6). From Eqs. 8, 9:

$$
\frac{q_{o}}{p_{o}}=\left(\frac{Q}{P}\right)^{1 / 3}\left(\frac{G_{*}}{E_{*}}\right)^{2 / 3} \geq \mu \quad \text { i. e. } \quad \frac{Q}{P} \geq 4\left(1+v_{*}\right)^{2} \mu^{3} \equiv N
$$

Thus, for $N \leq Q / P \leq M$, localized micro-slip occurs within a disk of radius $c$ in the plane of the contact surface so that $q(c)=\mu p(c)$ over its circumference. From Eqs. 8 and 9:

$$
\frac{q(c)}{p(c)}=\frac{G_{*}}{E_{*}} \sqrt{\frac{a^{2}-c^{2}}{\alpha^{2}-c^{2}}}=\mu \text { which results in } c=\sqrt{\frac{a^{2}-\left[2\left(1+v_{*}\right) \mu\right]^{2} \alpha^{2}}{1-\left[2\left(1+v_{*}\right) \mu\right]^{2}}} \text { (Eq. 18) }
$$

In this model step, the volumetric shear work $W_{i t}=q_{i}^{2} / 2 G_{*}=U_{t} / V$ stored over the disk of radius $a$ in the ideal elastic step (Fig. 4b, area OBI) is recast and equated to the actual shear work (area OADH). The ideal shear stress $q_{i}$ saturates at the friction limit $\mu p$, and the ideal displacement $\delta_{i}$ is extended to $\delta$, to absorb the excess work $W_{i r}$ (area $\left.A B C=I C D H\right)$. Slip within the disk of radius $c$ releases volumetric work $W_{i f}$ (Fig. 4b, area OADF) dissipated as heat due to friction. Upon unloading, the volumetric elastic work $W_{u r}=W_{i t}-W_{i f}$ (Fig. $4 \mathrm{~b}$, area FDH) is in part released as kinetic energy $U_{\text {outt }}$ (area GJDH) to the particulate surroundings, and in part stored as volumetric elastic work $W_{r t}$ in the residual shear stress field at the contact (area FJG):

$$
\begin{gathered}
W_{i t}=\frac{1}{2 G_{*}}\left(\frac{2}{3} q_{o}\right)^{2}, \quad W_{u r}=\frac{1}{2 G_{*}}\left(\frac{2}{3} \mu p_{o}\right)^{2}=W_{i t} \zeta^{2}, \quad W_{i r}=\frac{1}{2 G_{*}}\left(\frac{2}{3}\left(q_{o}-\mu p_{o}\right)\right)^{2}=W_{i t}(1-\zeta)^{2} \\
W_{i f}=W_{i t}-W_{u r}=\frac{1}{2 G_{*}}\left[\left(\frac{2}{3} q_{o}\right)^{2}-\left(\frac{2}{3} \mu p_{o}\right)^{2}\right]=W_{i t}\left[1-\left(\frac{\mu p_{o}}{q_{o}}\right)^{2}\right]=W_{i t}\left(1-\zeta^{2}\right) \\
\text { where } \frac{\mu p_{o}}{q_{o}}=\mu \frac{P}{Q}\left(\frac{a}{\alpha}\right)^{2} \equiv \zeta \quad \text { (Eq. 20) }
\end{gathered}
$$

When $\zeta \geq 1 / 2$, then no slip or only partial micro-slip within radius $c$ occurs, and the contact sides are attached. In this case, and upon unloading of the contact surface, the volumetric elastic work $W_{u r}$ (Fig. $4 \mathrm{~b}$, area FDH) is in part released as kinetic energy $U_{\text {outt }}$ to the particulate surroundings (area GJDH), and in part stored as volumetric shear elastic work $W_{r t}$ (area FJG):

$$
W_{r t}=W_{i r}=W_{i t}(1-\zeta)^{2}, \quad U_{\text {outt }} / V=W_{u r}-W_{i r}=W_{i t}(2 \zeta-1)
$$

When $\zeta<1 / 2$, then full slip occurs over the contact radius, and the contact sides are detached. In this case, upon unloading, all the volumetric elastic work $W_{u r}$ remains stored as residual field $W_{r t}$ in the contact, while no kinetic energy $U_{\text {outt }}$ is released to the surroundings:

$$
W_{r t}=W_{u r}=W_{i t} \zeta^{2}, \quad U_{o u t t} / V=0
$$

\section{$\underline{2.5 \text { Contact Surface Yield and Joint }}$}


During the loading phase and in its subsequent inelastic step, the model detects if the two elastic field components, i.e. the ideal volumetric pressure work $W_{i n}=p_{o}^{2} / 2 E_{*}=U_{n} / V$ and the residual shear work $W_{r t}=$ $q_{o}^{\prime}{ }_{0}^{2} / 2 G_{*}$ (Eq. 21 or 22 ) result into equivalent stress levels $\sigma(r)$ causing plastic yield at the contact surface. According to the maximum distortion energy criterion (von Mises) [31-33], at the axis of the contact disk:

$$
\sigma(0)=\sqrt{p_{o}^{2}+3 q_{o}^{\prime 2}} \geq \sigma_{y}^{*} \text { where } q_{o}^{\prime}=\sqrt{2 W_{r t} G_{*}} \text { and } \sigma_{y}^{*}=\eta \frac{2 \sigma_{y 1} \sigma_{y 2}}{\sigma_{y 1+} \sigma_{y 2}}
$$

The equivalent yield stress of the contact $\sigma_{y}^{*}$ accounts for the yield stresses $\sigma_{y 1}, \sigma_{y 2}$ of the contacting materials. The yield coefficient $\eta$ incorporates the effect of contact surface conditions (roughness, surface layers, etc.) on local yield of the contact and, therefore, controls its joining status as described below.

In this case, plastic yield occurs within a disk of radius $\kappa$ (Fig. 7a), defined implicitly by:

$$
\kappa: \quad \sigma(\kappa)=\sqrt{p^{2}(\kappa)+3 q^{\prime 2}(\kappa)}=\sigma_{y}^{*}(\text { Eq. 24) }
$$

The equivalent compressive stress level $\sigma(r)$ is given by (Fig. 7a):

$$
\begin{gathered}
\sigma(r)=\sigma_{y}^{*}+\frac{E_{*}^{\prime}}{E_{*}}\left(\sqrt{p^{2}(r)+3 q^{\prime 2}(r)}-\sigma_{y}^{*}\right) \text { for } 0 \leq r \leq \kappa \text { (yielded disk) (Eq. 25) } \\
\sigma(r)=\sqrt{p^{2}(r)+3 q^{\prime 2}(r)} \text { for } \kappa \leq r \leq \alpha \quad \text { (elastic annulus) (Eq. 26) }
\end{gathered}
$$

where the equivalent strain-hardening elastic modulus $E_{*}^{\prime}$ is defined similar to Eq. 6 .

Contact unloading is modeled by superposition of an opposite elastic distribution $-\sigma(r)-\sigma_{r}(r)$ (Fig. 7b). When a joint is formed at the contact, a residual elastic stress field $\sigma_{r}(r)$ develops, with a tensile elastic distribution over the disk of radius $\kappa$, and a compressive elastic distribution over the annulus of radius $\kappa<r<\alpha$, balancing each other. When a joint is not formed, the unbalanced tensile and compressive distributions result in a net elastic load separating the contact.

In the subsequent inelastic step of the model, the volumetric elastic work $W_{\text {in }}=\left(U_{\text {in }}-U_{\text {outt }}\right) / V-W_{\text {if }}-W_{r t}$, stored as ideal normal stress field $\sigma_{i}$ over the contact of radius $\alpha$ (Fig. 4a, area OBI), is recast and equated to the actual volumetric work (area OADH) during the loading phase. During this step, elastoplastic strain-hardening to actual stress $\sigma$ (Eq. 25) occurs within the disk $\kappa$, and ideal strain $\varepsilon_{i}$ is extended to actual $\varepsilon$, to absorb the excess work $W_{\text {ir }}$ (area $\left.A B C=I C D H\right)$. By equating the ideal elastic with the actual volumetric work areas and by similarity of triangles (Fig. 4a):

$$
\begin{gathered}
W_{\text {in }}=\frac{E_{*}}{2}\left(\frac{2}{3} \varepsilon_{i}\right)^{2}=\frac{E_{*}}{2}\left(\frac{2}{3} \varepsilon\right)^{2}-\frac{E_{*}-E_{*}^{\prime}}{2}\left[\frac{2}{3}\left(\varepsilon-\varepsilon_{y}\right)\right]^{2} \quad \varepsilon_{i}^{2}=\varepsilon^{2}-(1-b)\left(\varepsilon-\varepsilon_{y}\right)^{2} \text { with } b \equiv \frac{E_{*}^{\prime}}{E_{*}} \\
\varepsilon=\sqrt{\frac{1}{b}\left[\varepsilon_{i}^{2}+\left(\frac{1}{b}-1\right) \varepsilon_{y}^{2}\right]}-\left(\frac{1}{b}-1\right) \varepsilon_{y}(b \neq 0) \text { or } \varepsilon=\frac{\varepsilon_{i}^{2}+\varepsilon_{y}^{2}}{2 \varepsilon_{y}}(b=0) \quad \text { (Eq. 28) } \\
\sigma=\sigma_{y}^{*}+E_{*}^{\prime}\left(\varepsilon-\varepsilon_{y}\right) \text { and } \sigma^{\prime}=E_{*} \varepsilon \quad \text { (Eq. 29) }
\end{gathered}
$$


Plastic yield within the disk of radius $\kappa$ releases volumetric deformation work $W_{i p}$ (Fig. 4a, area OADF) dissipated as heat. Therefore, unloading of the contact leaves a balance of volumetric elastic work $W_{u r}=W_{i n}-W_{i p}$ (area FDH). By geometric calculations on similar triangles in Fig. 4a:

$$
\begin{gathered}
W_{i r}=W_{i n}\left(\frac{\varepsilon}{\varepsilon_{i}}\right)^{2}\left(1-\frac{\sigma_{y}^{*}}{\sigma_{\prime}}\right)^{2}\left(1-\frac{E_{*}^{\prime}}{E_{*}}\right)\left(\frac{\sigma_{i}-\sigma_{y}^{*}}{\sigma^{\prime}-\sigma_{y}^{*}}\right)^{2}=W_{i n}(1-b)\left(1-\frac{\psi}{\xi}\right)^{2} \\
W_{u r}=W_{i n}\left(\frac{\varepsilon}{\varepsilon_{i}}\right)^{2}\left[\frac{\sigma_{y}^{*}}{\sigma_{\prime}}+\left(1-\frac{\sigma_{y}^{*}}{\sigma_{\prime}}\right) \frac{E_{*}^{\prime}}{E_{*}}\right]^{2}=W_{i n}\left[\frac{\psi}{\xi}\left(1-b+\frac{b}{\psi}\right)\right]^{2} \\
W_{i p}=W_{i n}-W_{u r}=W_{i n}\left\{1-\left[\frac{\psi}{\xi}\left(1-b+\frac{b}{\psi}\right)\right]^{2}\right\}
\end{gathered}
$$

where $\xi=\frac{\varepsilon_{i}}{\varepsilon}=\frac{\sigma_{i}}{\sigma^{\prime}}, \quad \psi=\frac{\varepsilon_{y}}{\varepsilon}=\frac{\sigma_{y}^{*}}{\sigma^{\prime}}$ and (from Eq. 27) $\xi^{2}=1-(1-b)(1-\psi)^{2}$

Upon unloading, when $W_{u r} \geq W_{i r}, W_{u r}$ is in part stored as volumetric elastic work $W_{r n}$ in the residual normal stress field at the contact (area FJG), and in part released as kinetic energy $U_{\text {outn }}$ (area GJDH) to the particulate surroundings by the separating sides, and therefore no joint forms:

$$
\begin{gathered}
W_{r n}=W_{\text {ir }}=W_{\text {in }}(1-b)\left(1-\frac{\psi}{\xi}\right)^{2}, \\
U_{\text {outn }} / V=W_{\text {ur }}-W_{\text {ir }}=W_{\text {in }}\left\{\left[\frac{\psi}{\xi}\left(1-b+\frac{b}{\psi}\right)\right]^{2}-(1-b)\left(1-\frac{\psi}{\xi}\right)^{2}\right\}
\end{gathered}
$$

When $W_{u r}<W_{\text {ir }}$, no kinetic energy $U_{\text {outn }}$ is released to the surroundings and a joint forms at the contact. The entire volumetric elastic work $W_{u r}$ is stored in the residual normal stress field $W_{r n}$ at the joint area:

$$
W_{r n}=W_{u r}=W_{\text {in }}\left[\frac{\psi}{\xi}\left(1-b+\frac{b}{\psi}\right)\right]^{2}, \quad U_{\text {outn }} / V=0
$$

For such a developed joint, its normal and shear strength to subsequent collision events are described respectively by the externally supplied volumetric elastic works $W_{j n}, W_{j t}$ necessary to separate the joint. These terms, together with the previously stored residual fields $W_{r n}, W_{r t}$, are required to reverse the plastic deformation $W_{i p}$ and friction work $W_{\text {if }}$ necessary to split the bonded contact:

$$
W_{j n}=W_{i p}+W_{i f}-W_{r n}, W_{j t}=W_{i p}+W_{i f}-W_{r t}
$$

Therefore, a joint can be broken upon a subsequent impact by developing a tensile $W_{i n}>W_{j n}$ or a shear $W_{i t}^{\prime}>W_{j t}$ elastic field exceeding the respective joint strength at the joined contact. In this case, the stress distributions at the separating contact surface are wiped out, while internal residual elastic fields may be released as kinetic energy to the surroundings. The total elastic energy $U_{r}$ stored in the residual stress field at the contact, the total plastic deformation $U_{p}$ and friction energy $U_{f}$ converted into heat $H$, the total joint bonding energy $B_{j}$ and the total kinetic energy released to the surroundings $U_{\text {out }}$ are:

$$
\begin{array}{r}
U_{p}=W_{i p} V, \quad U_{f}=W_{i f} V, \quad U_{r}=\left(W_{r n}+W_{r t}\right) V, \\
B_{j}=U_{p}+U_{f}-U_{r}, \quad U_{\text {out }}=U_{\text {outn }}+U_{\text {out }}, \quad H=U_{p}+U_{f}
\end{array}
$$

\section{$\underline{2.6 \text { Surface and Bulk Deformation }}$}


The loads $P_{j} Q_{j}$ in the analysis above are determined for all contacts $j=1 . . . N$ of the examined particulate, with either a colliding impactor (Eq. 13) or an external particulate (Eq. 12) of the adjacent layer, along its boundary $L$ (Fig. 3). The latter contacts may generate or break boundary joints with adjacent clusters and particles, leading to their potential integration to or separation from the examined particulate during the process. The boundary loading is also necessary to determine internal loads among, and deformations of, the WE primitives $i=1 . . . n$ in the bulk of the particulate, as its structure evolves. Thus, along with contact stiffnesses $K_{n j} K_{t j}$ at the boundary contacts, computation of boundary displacements $\beta_{j}, \delta_{j}$ is also required. This, in turn, requires the reaction load distributions (and therefore the distribution of stiffnesses and displacements) in the "surrounding medium" matter (Fig. 3). Contact with this medium is assumed elastic since, if it were locally inelastic and potentially subject to yield and joining with the particulate, this contacting component would have been included as part of the adjacent layer. The effective boundary stiffnesses are:

$$
K_{n}^{*}=\frac{1}{L} \sum_{j=1}^{N} K_{n j} \alpha_{j}=\frac{1}{L} \sum_{j=1}^{N} 2 E_{* j} R_{* j} \beta_{j}, \quad K_{t}^{*}=\frac{1}{L} \sum_{j=1}^{N} K_{t j} a_{j}=\frac{1}{L} \sum_{j=1}^{N} 2 G_{* j} R_{* j} \delta_{j}
$$

To avoid the computational cost of this redundant (statically indeterminate), probabilistic configuration of discrete random particulate matter [34,35], the model instead adopts a uniform, homogeneous and isotropic continuum as "surrounding medium" to the particulate, absorbing elastic energy equivalent to that of the discrete matter. In the initial ideal model step, this Eshelby surrounding medium is assumed linear elastic with unipolar normal loading in compression only, reflecting the nature of the original particulate matter. This hollow medium acts as a distributed spring in parallel to the stiffness of the examined particulate it surrounds, because of their common displacements at their contact boundary. This stiffness in parallel at the boundary can be replaced by an equivalent one resulting from a conceptual "overlapping medium", inscribed to the examined particulate volume. This solid medium is conformal to the particulate and is assumed of general ellipsoidal form (Figs. 2, 3). The 3D boundary value problem of the internal elastic field in such an ellipsoidal medium generated by a reaction load distribution on its boundary is solved in the literature [36-44]. The requisite elastic moduli $E_{0}, G_{o}$ of the medium are derived through particulate-medium boundary displacement compatibility $(\beta, \delta)$ from the residual elastic field work $W_{r i}$ over volume $V_{i}$ and kinetic energy released to the surrounding $U_{\text {outi }}$ for all WE contacts $i=1 . . . n$ in the particulate (Eqs. 19-22, 30-35):

$$
\begin{aligned}
\sum_{i=1}^{n} W_{r n i} V_{i} & \equiv \frac{1}{2} \sum_{i=1}^{n} E_{i} V_{i} \beta^{2}, \sum_{i=1}^{n} U_{\text {outni }} \equiv \frac{1}{2} E_{o} V_{o} \beta^{2} & E_{o}=\frac{\sum_{i=1}^{n} E_{i} V_{i}}{V_{o}} \frac{\sum_{i=1}^{n} U_{\text {outni }}}{\sum_{i=1}^{n} W_{\text {rni }} V_{i}} \\
\sum_{i=1}^{n} W_{r t i} V_{i} & \equiv \frac{1}{2} \sum_{i=1}^{n} G_{i} V_{i} \delta^{2}, \sum_{i=1}^{n} U_{\text {outt } i} \equiv \frac{1}{2} G_{o} V_{o} \delta^{2} & G_{o}=\frac{\sum_{i=1}^{n} G_{i} V_{i}}{V_{o}} \frac{\sum_{i=1}^{n} U_{\text {outti }}}{\sum_{i=1}^{n} W_{r t i} V_{i}}
\end{aligned}
$$

where the medium volume $V_{o}=\Sigma V_{i}$ for a fully densified BM particulate.

Using the boundary reaction conditions above, the model in its initial elastic step determines the ideal deformations of all ellipsoidal primitives (WEs) inside the particulate, using Castigliano's theorem of least work (strain energy method) [26]. This is done by applying fictitious incremental normal and shear test loads $p_{k} q_{k}$, e.g. at the four semi-axis endpoints on the WE boundary $(k=1 . . .4$, Fig. 2$)$, and by calculating the strain energy $U_{i}$ stored in the WE $(i=1 \ldots n)$. The WE is loaded in pressure and shear by $P_{j}$, $Q_{j}$ respectively at its contact points with normal and shear stiffnesses $K_{n j}, K_{t j}\left(j=1 \ldots \mathrm{N}\right.$, Eq. 12) at angles $\varphi_{\mathrm{jk}}$ and distances $l_{\mathrm{jk}}$ with respect to the test loads (Fig. 2). According to Castigliano's theorem, the normal and shear deflections $\beta_{k}, \delta_{k}$ at the test points are determined via the elastic energy increments as: 


$$
\begin{gathered}
\beta_{i k}=\lim _{p_{i k}=0} \frac{\partial U_{n i k}}{\partial p_{i k}}, \delta_{i k}=\lim _{q_{i k}=0} \frac{\partial U_{t i k}}{\partial q_{i k}} \quad \text { where } \\
U_{n i k}=\sum_{j} W_{r n i j} V_{i}+\frac{\sqrt{2} l_{i x} p_{i k}^{2}}{\pi\left(E_{i}+E_{o}\right) l_{i y} l_{i z}}+\sum_{j}\left[\frac{P_{i j} p_{i k} \cos \varphi_{j k}}{K_{n i j} \cos \varphi_{j k}+\pi\left(E_{i}+E_{o}\right) l_{j k}}+\frac{Q_{i j} p_{i k} \sin \varphi_{j k}}{K_{t i j} \sin \varphi_{j k}+\pi\left(E_{i}+E_{o}\right) l_{j k}}\right] \\
U_{t i k}=\sum_{j} W_{r t i j} V_{i}+\frac{\sqrt{2} l_{i y} q_{i k}^{2}}{\pi\left(G_{i}+G_{o}\right) l_{i x} l_{i z}}+\sum_{j}\left[\frac{Q_{i j} q_{i k} \cos \varphi_{j k}}{K_{t i j} \cos \varphi_{j k}+\pi\left(G_{i}+G_{o}\right) l_{j k}}+\frac{P_{i j} q_{i k} \sin \varphi_{j k}}{K_{n i j} \sin \varphi_{j k}+\pi\left(G_{i}+G_{o}\right) l_{j k}}\right]
\end{gathered}
$$

In Eqs. 42 and 43, the residual elastic energies at the contact points (first terms) and the strain energies stored by the test loads alone (second terms) do not contribute to the partial derivatives of Eq. 41, which therefore depend only on the coupling work performed by contact loads over their displacements due to the test loading (third terms).

Next, in the inelastic step of the model, the ideal elastic deformation energies above (Fig. 4, area OBI) are recast and equated to the actual displacement work with friction slip and plastic yield (area OADH), with reduction of the respective loads and extension of displacements to absorb excess work (area $A B C=I C D H)$. First, to compensate for frictional work losses at the boundary with and inside the surrounding medium, assumed to have same slip properties as the particulate, the ideal elastic energies (Eqs. 42,43 ) are modified as:

$$
U_{i k}^{\prime}=U_{i k}\left[1-\frac{\sum_{i=1}^{n} U_{o u t t i}}{\sum_{i=1}^{n} U_{i n i}}\left(1-\zeta_{i k}^{2}\right)\right] \text { where } \zeta_{i k}=\mu_{k} 2\left(1+v_{* i}\right) \frac{\alpha i_{k}}{a_{i k}} \frac{\beta_{i k}}{\delta_{i k}} \quad \text { at the boundary (Eq. 44) }
$$

Subsequently, the contact slip and friction calculations on deformations are performed similar to Eqs. $16-22$, e.g. for contact displacement $\delta_{k}$ :

$$
\delta_{k}=\frac{\delta_{i k}^{2}+\delta_{\mu}^{2}}{2 \delta_{\mu}}
$$

where $\delta_{\mu}$ is the contact displacement at the onset of frictional slip (Fig. 4b).

Second, the contact yield and joint energetics calculations are carried out similar to Eqs. 23-35 (Fig. 4a). The resulting normal and shear bonding energies at contact joints allow for reassessment of their integrity or separation (Eq. 36), and kinetic energy and heat released to the environment of the particulate are determined (Eq. 37). New contact areas may establish additional joints in the particulate and its boundary with adjacent clusters and particles, therefore gradually augmenting its size. Finally, the previously computed displacements $\beta_{k}$ and $\delta_{k}(k=1 \ldots 4$, Eqs. 38-46) at the semi-axis endpoints of each WE domain are used to update its geometric features $\left(X, Y ; \phi_{x}, \phi_{y} ; I_{x} I_{y} ; R_{x}, R_{y}\right)$. Therefore, the internal structure of the examined particulate evolves over time by deformation of its constituent WE primitives.

\section{Model Results}

\section{$\underline{3.1 \text { Calibration Tests on Foils }}$}

Laboratory testing of the model is performed on a low-energy planetary BM system (Fritsch Mono Mill Pulverisette 6 ) in nitrogen inert atmosphere, with five balls of $R_{\text {ball }}=5 \mathrm{~mm}$ radius in a rounded cylindrical $80 \mathrm{ml}$ vial rotating at $300 \mathrm{rpm}$ and made of stainless steel. The bimetallic Al-Ni reactive system is employed for model calibration and validation, with material properties shown in Table 1 [45], and with ball energetics described by $s=1 \mathrm{~m} / \mathrm{s}$ in Eq. 1 (Fig. 5). In addition, the contact surface parameters, i.e. the friction coefficient $\mu$ (Eqs. 16-22) and yield coefficient $\eta$ (Eqs. 23-26) depend sensitively on material 
surface state and process conditions, and are experimentally calibrated. Instead of metal powders, for modeling simplicity and experimental repeatability, multilayer sandwiches of alternating bilayers of $5 \mathrm{Al}$ layers (each of thickness $h_{A L}=200 \mu \mathrm{m}$, consisting of 4 foils $50 \mu \mathrm{m}$ thick) and $6 \mathrm{Ni}$ foils (of thickness $h_{N I}=50$ $\mu \mathrm{m})$ are assembled as in Fig. 8. Each assembly is fitted tightly to the cylindrical vial wall covering its full internal surface, therefore corresponding to deterministic WE parameters $I_{x}=\pi R_{\text {vial }} I_{y}=h / 2, R_{x}=R_{\text {vial }}, R_{y}=0$. After processing for $1,2 \ldots 6 \mathrm{~min}$ the ball-milled assemblies are length-wise sliced into 5 strips (of width $w=10 \mathrm{~mm}$ each) as samples.

Table 1: Ball-milled and impactor material properties used in model simulations.

\begin{tabular}{|l|l|l|l|}
\hline Material/Properties & Aluminum (99.5\%) & Nickel (99.95\%) & Stainless Steel (1.4404) \\
\hline Density $\rho\left(\mathrm{kg} / \mathrm{m}^{3}\right)$ & 2700 & 8908 & 7955 \\
\hline Elastic Modulus $E(\mathrm{GPa})$ & 70 & 170 & 197 \\
\hline Shear Modulus $G(\mathrm{GPa})$ & 26 & 65 & 77.6 \\
\hline Poisson Ratio $v$ & 0.35 & 0.31 & 0.27 \\
\hline Strain hardening Modulus $E^{\prime}(\mathrm{GPa})$ & 3.5 & 5 & 9.5 \\
\hline Yield Tensile Stress $\sigma_{y}(\mathrm{MPa})$ & 17.5 & 140 & 290 \\
\hline Yield Shear Stress $\tau_{y}(\mathrm{MPa})$ & 9.6 & 119 & 275 \\
\hline Ultimate Tensile Strength $\sigma_{u}(\mathrm{MPa})$ & 45 & 167 & 580 \\
\hline Ultimate Shear Strength $\tau_{u}(\mathrm{MPa})$ & 29 & 150 & 522 \\
\hline
\end{tabular}

Each sample is subjected to progressive steady tension until complete shearing of the bilayer joints on a universal testing machine (Instron 4505). For each sample, the total debonding work $B$ is measured through the area under the load-displacement curve until failure (comparable to the solid line in Fig. 4) and plotted on Fig. 9. The figure shows an approximately linear increase of sample bond energy with time during the very first BM stages with quite sparse, mostly non-overlapping interface joints over the bilayer section of Fig. 8. This result is compared to the model-predicted total joint bonding energy $\Sigma B_{j}$ of all joints $j$ formed across the bilayer sandwich (Eq. 37). In Fig. 9, the initial surface parameter estimates $\mu=0.32, \eta=1$ lead to under-prediction of the tensile data, presumably because of underestimated frictional and plastic yield effects in heating and softening of the initial materials at room temperature, thus advancing contact surface joining. The two parameters are subsequently calibrated by minimization of the rms error of trial model predictions with respect to the laboratory measurements in Fig. 9, leading to eventual calibrated values $\mu=0.39, \eta=0.81$.

Fig. 10 shows the micrograph of a representative sample cross section of the resulting foil particulate microstructure in the Al-Ni multilayer sandwich, with layers shown separated by shearing, after 3 min of BM processing. The layer of four Al foils on the left appears to have progressed in local in-plane deformations (with concomitant alterations of their original thickness), and in monometallic bonding between the foils, presumably because of the lower yield point of Al. The Ni foil on the right exhibits only minor deformation and sparse bimetallic joints with the Al layer. This is attributable to the higher value of equivalent yield stress at the Al-Ni contact boundary (Eq. 23), and thus the lower probability for sufficient bonding work (Eqs. 36, 37) to be supplied by ball impact energy distribution (Eqs. 1, 3).

\section{$\underline{3.2 \text { Validation Tests on Powders }}$}

Validation of the model with the previously calibrated surface parameters, material and energetic properties is performed experimentally at the process conditions above, using Al and Ni powders (Alfa 

intermetallic compound), and total weight of $32 \mathrm{~g}$. The BM run is interrupted every $2 \mathrm{~h}$, and during each interval $2 \mathrm{~g}$ of the sample material is removed for structural observation [46]. To avoid exposure to air the sample removal procedure is conducted in a nitrogen-filled glove box. Particulate samples weighing $0.5 \mathrm{~g}$ are compacted under a pressure of $51 \mathrm{MPa}$ into disk pellets, $10 \mathrm{~mm}$ in diameter and $1.3 \mathrm{~mm}$ in thickness, to facilitate handling during their analysis by scanning electron microscopy (SEM, Tescan Vega LSU). Implementation of the integrated model following the previous run-time mechanics equation steps and using off-line pre-calculated look-up tables for their energetics, is performed on a standard numerical platform on a desktop computer at 42 GFLOPS as in [22]. The model achieves real-time execution speeds up to about $12 \mathrm{~h}$ of BM time, but runs behind the laboratory process thereafter due to the increasing complexity of the growing particulate under consideration.

The SEM micrographs along with the model-simulated microstructures of ball-milled samples for 6,12 , 18 and $22 \mathrm{~h}$ are shown in Fig. 11 (Al-dark phase, Ni-light phase). The figure shows progressive evolution of a globular (Apollonian pack) aggregation of harder, initially undeformed Ni particles with intermediate softer, gradually deforming Al particles, into a fully-intermixed lamellar Ni-Al branching network. Deformation of particulates and their constituent particle WE domains to lower aspect ratio (i.e. flatter) profiles is due to compressive loading by impactors during BM. Intermixing of the resulting lamellae is observed, primarily because of shear loading at their contact boundaries. Coagulation and spheronization of domain boundaries results from interfacial friction, while plastic yield also contributes to thermal softening and bonding of their surfaces. Micro-weld joints explain the formation of curled eddy-like micropatterns locally requiring interfacial tension to maintain material cohesion. Fragmentation of Ni particles and gradual hardening of the Al interphase due to geometric confinement, strain and interdiffusion effects, potentially because of solid solution interlayers developing at the contact surfaces with increasing temperature, are also observed in the micrographs.

The similarity of geometrical attributes between experimental and simulated sections is quantified by comparing their fractal dimension components [47], computed by image processing software through box-counting algorithms [48]. In Table 2, the modelled section parameters deviate in the range of $+3.6 \%$ to $-3.0 \%$ from those of the laboratory micrographs. This could be attributable in part to uncertainty in geometrical, material and process conditions, along with potential drift over time of the calibrated contact surface parameters.

Table 2: Fractal dimension parameters for laboratory and simulated microstructures during BM process.

\begin{tabular}{|l|l|l|l|l|l|l|l|l|}
\hline \multirow{2}{*}{$\begin{array}{c}\text { BM Time/ } \\
\text { Fractal Dimension }\end{array}$} & \multicolumn{2}{c|}{6 Hours } & \multicolumn{2}{c|}{12 hours } & \multicolumn{2}{c|}{18 hours } & \multicolumn{2}{c|}{22 hours } \\
\cline { 2 - 10 } & Exper & Model & Exper & Model & Exper & Model & Exper & Model \\
\hline Capacity Dimension & 2.53 & 2.62 & 2.65 & 2.72 & 2.78 & 2.83 & 2.54 & 2.60 \\
\hline Information Dimension & 2.43 & 2.49 & 2.55 & 2.60 & 2.75 & 2.77 & 2.49 & 2.51 \\
\hline Correlation Dimension & 2.35 & 2.28 & 2.42 & 2.37 & 2.72 & 2.64 & 2.44 & 2.38 \\
\hline Probability Dimension & 2.41 & 2.46 & 2.43 & 2.47 & 2.49 & 2.48 & 2.41 & 2.45 \\
\hline
\end{tabular}

\section{Conclusions}


The computational model established in this article introduces several original contributions to the wealthy literature of MA simulation. These include: the warped ellipsoid (WE) as universal domain primitive, from spheroidal powders to lamellar particulate layers; implementation of experimentally calibrated kinetic theory-based motion of the ball impactors; ideal elastic-to-inelastic dissipative energetic material transformations of surface friction slip and bulk Castigliano deformation; particulate boundary conditions by Eshelby equivalent surrounding/overlapping inscribed media; energy-based mechanics modeling synthesis, enabling real-time computational performance; calibration of frictional and deformation parameters via simple BM foil tensile tests; and ability of the random chaotic model dynamics to predict evolution of the full fractal structure of BM particulates.

The reported morphological correlation of the simulated structural evolution with that of experimental samples for both bimetallic powder particulates and multilayer foils appears to support the fundamental underpinnings of the model. In particular, the WE primitive constituents of BM structures offer flexibility in cross-sectional profile modeling across the 2D foil - 3D particulate range, while lending themselves to simple, computationally-efficient semi-analytical geometry representation and transformation processing. Moreover, the concept of gradual surface and bulk displacements in two distinct stages, i.e. an ideal linear elastic step followed by an inelastic frictional slip and plastic yield stage, offers insights and decouples the mechanics of different material phenomena without loss of generality. Last, the energy-based approach to deformation and joining of BM structures maintains computationally simple and universal scalar metrics and criteria for the onset of nonlinear effects.

Residual deviations of the model predictions from laboratory observations, aside from parameter discrepancies, appear to stem mainly from the assumptions for isothermal, compositionally-invariant material processing. As discussed in multilayer foil bonding energies, heat released via friction and plasticity may create local thermal impact during collisions, upsetting thermal equilibrium and leading into sharp temperature gradient-driven conductive transfer, affecting material properties and surface energies. It also provides activation to homogeneous and heterogeneous diffusion across domain surfaces, promoting contact joining, composition gradients and solid solution zones. In conjunction with temperature increase, the latter drive solid-state intermetallic reactions near the boundaries, especially during the later stages of the BM process [46].

Further research is therefore in progress to complement the presented mechanics formulation with a thermal/compositional model. However, the resulting additional complexity and computational cost may hamper run-time efficiency of the model for its intended use as a real-time observer of the BM process. Because of its closed batch configuration and kinematics preventing direct sensing and feedback of internal process variables, such an observer could assist closed-loop control of the structure and properties of the BM product. The optimal compromise between model accuracy and efficiency is currently under study and will be reported separately.

\section{Acknowledgment}

This research was supported in part by a Khalifa University Internal Research Fund (Level 1) award and by the US Department of Energy, National Nuclear Security Administration under Award Number DENA0002377. KU students Khatera Farzanah, Mira Hassan and Rauda Al Mheiri are gratefully acknowledged for laboratory work in calibration tests. The authors also wish to thankfully acknowledge particularly constructive suggestions on this article context and references by one anonymous reviewer. 


\section{$\underline{\text { References }}$}

[1] A.S. Rogachev, A.S. Mukasyan, Combustion of heterogeneous nanostructural systems (review), Combust. Explos. Shock Waves. 46 (2010) 243-266. doi:10.1007/s10573-010-0036-2.

[2] Z. Gu, Q. Cui, J. Chen, J. Buckley, T. Ando, D. Erdeniz, P. Wong, A. Hadjiafxenti, P. Epaminonda, I.E. Gunduz, C. Rebholz, C.C. Doumanidis, Fabrication, characterization and applications of novel nanoheater structures, Surf. Coatings Technol. 215 (2013) 493-502. doi:10.1016/j.surfcoat.2012.06.095.

[3] K. V. Manukyan, B.A. Mason, L.J. Groven, Y.C. Lin, M. Cherukara, S.F. Son, A. Strachan, A.S. Mukasyan, Tailored reactivity of Ni+Al nanocomposites: Microstructural correlations, J. Phys. Chem. C. 116 (2012) 21027-21038. doi:10.1021/jp303407e.

[4] A. Hadjiafxenti, I.E. Gunduz, T. Kyratsi, C.C. Doumanidis, C. Rebholz, Exothermic reaction characteristics of continuously ball-milled Al/Ni powder compacts, Vacuum. 96 (2013) 73-78. doi:10.1016/j.vacuum.2013.03.004.

[5] A. Hadjiafxenti, I.E. Gunduz, C.C. Doumanidis, C. Rebholz, Spark ignitable ball milled powders of Al and Ni at NiAl composition, Vacuum. 101 (2014) 275-278. doi:10.1016/j.vacuum.2013.09.006.

[6] D.R. Maurice, T.H. Courtney, The physics of mechanical alloying: A first report, Metall. Trans. A. 21 (1990) 289-303. doi:10.1007/BF02782409.

[7] D. Maurice, T.H. Courtney, Modeling of mechanical alloying: Part I. deformation, coalescence, bdand fragmentation mechanisms, Metall. Mater. Trans. A. 25 (1994) 147-158. doi:10.1007/BF02646683.

[8] D. Maurice, T.H. Courtney, Modeling of mechanical alloying: Part II. Development of computational modeling programs, Metall. Mater. Trans. A. 26 (1995) 2431-2435. doi:10.1007/BF02671257.

[9] D. Maurice, T.H. Courtney, Milling dynamics: Part III. integration of local and global modeling of mechanical alloying devices, Metall. Mater. Trans. A. 27 (1996) 1981-1986. doi:10.1007/BF02651947.

[10] T.H. Courntey, Process Modelling of Mechanical Alloying (Overview), Mater. Trans. JIM. 36 (1995) 110-122. https://www.jstage.jst.go.jp/article/matertrans1989/36/2/36_2_110/_pdf.

[11] P. Le Brun, L. Froyen, L. Delaey, The modelling of the mechanical alloying process in a planetary ball mill: comparison between theory and in-situ observations, Mater. Sci. Eng. A. 161 (1993) 7582. doi:10.1016/0921-5093(93)90477-V.

[12] M. Abdellaoui, E. Gaffet, The physics of mechanical alloying in a planetary ball mill: Mathematical treatment, Acta Metall. Mater. 43 (1995) 1087-1098. doi:10.1016/0956-7151(95)92625-7.

[13] M. Abdellaoui, E. Gaffet, The physics of mechanical alloying in a modified horizontal rod mill: Mathematical treatment, Acta Mater. 44 (1996) 725-734. doi:10.1016/1359-6454(95)00177-8.

[14] M. Magini, A. lasonna, F. Padella, Ball milling: An experimental support to the energy transfer 

3.

[15] A. Iasonna, M. Magini, Power measurements during mechanical milling. An experimental way to investigate the energy transfer phenomena, Acta Mater. 44 (1996) 1109-1117. doi:10.1016/1359-6454(95)00226-X.

[16] F. Delogu, R. Orrù, G. Cao, A novel macrokinetic approach for mechanochemical reactions, Chem. Eng. Sci. 58 (2003) 815-821. doi:10.1016/S0009-2509(02)00612-7.

[17] F. Delogu, G. Mulas, L. Schiffini, G. Cocco, Mechanical work and conversion degree in mechanically induced processes, Mater. Sci. Eng. A. 382 (2004) 280-287. doi:10.1016/j.msea.2004.05.047.

[18] S. Garroni, S. Enzo, F. Delogu, Mesostructural refinement in the early stages of mechanical alloying, Scr. Mater. 83 (2014) 49-52. doi:10.1016/j.scriptamat.2014.04.007.

[19] S. Garroni, S. Soru, S. Enzo, F. Delogu, Reduction of grain size in metals and metal mixtures processed by ball milling, Scr. Mater. 88 (2014) 9-12. doi:10.1016/j.scriptamat.2014.06.012.

[20] H. Kruggel-Emden, E. Simsek, S. Rickelt, S. Wirtz, V. Scherer, Review and extension of normal force models for the Discrete Element Method, Powder Technol. 171 (2007) 157-173. doi:10.1016/j.powtec.2006.10.004.

[21] P. Baláž, M. Achimovičová, M. Baláž, P. Billik, Z. Cherkezova-Zheleva, J.M. Criado, F. Delogu, E. Dutková, E. Gaffet, F.J. Gotor, R. Kumar, I. Mitov, T. Rojac, M. Senna, A. Streletskii, K. WieczorekCiurowa, Hallmarks of mechanochemistry: from nanoparticles to technology., Chem. Soc. Rev. 42 (2013) 7571-637. doi:10.1039/c3cs35468g.

[22] C.C. Doumanidis, I.E. Gunduz, C. Rebholz, C.C. Doumanidis, Real-Time Computational Model of Ball-Milled Fractal Structures, J. Nanotechnol. Eng. Med. 6 (2016) 31001. doi:10.1115/1.4031276.

[23] C.C. Doumanidis, H.A. Al Kaabi, A.S.M. Alzaabi, I.E. Gunduz, C. Rebholz, C.C. Doumanidis, Brownian-like kinematics of ball milling for particulate structural modeling, Powder Technol. 301 (2016) 1077-1084. doi:10.1016/j.powtec.2016.07.033.

[24] A.S. Rogachev, N.F. Shkodich, S.G. Vadchenko, F. Baras, D.Y. Kovalev, S. Rouvimov, A.A. Nepapushev, A.S. Mukasyan, Influence of the high energy ball milling on structure and reactivity of the Ni+Al powder mixture, J. Alloys Compd. 577 (2013) 600-605. doi:10.1016/j.jallcom.2013.06.114.

[25] J.R. Forshaw, A.G. Smith, Dynamics and Relativity, 2009. doi:10.1017/СВ09781107415324.004.

[26] S. Timoshenko, J. Goodier, Theory of Elasticity, McGraw-Hill, New York, 1970.

[27] A. Sackfield, D.A. Hills, Some useful results in the classical Hertz contact problem, J. Strain Anal. Eng. Des. 18 (1983) 101-105. doi:10.1243/03093247V182101.

[28] K.L. Johnson, Contact Mechanics, Cambridge University Press, Cambridge, 1985. doi:10.1017/СBO9781139171731.

[29] J. a Williams, R.S. Dwyer-Joyce, Contact Between Solid Surfaces, Mod. Tribol. Handb. 1 (2001) 121-162. doi:doi:10.1201/9780849377877.ch3\n10.1201/9780849377877.ch3.

[30] V.L. Popov, Rigorous Treatment of Contact Problems - Hertzian Contact, in: Contact Mech. Frict., Springer Berlin Heidelberg, Berlin, Heidelberg (2010) 55-70. doi:10.1007/978-3-642-10803-7_5.

[31] J. Jamari, D.J. Schipper, Plastic deformation and contact area of an elastic-plastic contact of ellipsoid bodies after unloading, Tribol. Int. 40 (2007) 1311-1318. doi:10.1016/j.triboint.2007.02.015.

[32] J.C. Chung, Elastic-plastic contact analysis of an ellipsoid and a rigid flat, Tribol. Int. 43 (2010) 491-502. doi:10.1016/j.triboint.2009.08.005.

[33] Q.J. Zheng, Z.Y. Zhou, A.B. Yu, Contact forces between viscoelastic ellipsoidal particles, Powder Technol. 248 (2013) 25-33. doi:10.1016/j.powtec.2013.03.020.

[34] J.L. Liou, C.M. Tsai, J.F. Lin, A microcontact model developed for sphere- and cylinder-based 
fractal bodies in contact with a rigid flat surface, Wear. 268 (2010) 431-442. doi:10.1016/j.wear.2009.08.033.

[35] I.C. Sinka, A model for the deformation of an ellipsoid subject to a large number of successive impacts with special reference to spheronisation, Powder Technol. 270 (2015) 592-598. doi:10.1016/j.powtec.2014.07.005.

[36] C. Chree, The Stresses and Strains in Isotropic Elastic Solid Ellipsoid in Equilibrium under Bodily Forces Derivable from a Potential of the Second Degree, Proc. R. Soc. London . 58 (1895) 39-59. doi:10.1098/rspl.1895.0004.

[37] J.D. Eshelby, The Determination of the Elastic Field of an Ellipsoidal Inclusion, and Related Problems, Proc. R. Soc. London. Ser. A. Math. Phys. Sci. 241 (1957) 376 LP-396. http://rspa.royalsocietypublishing.org/content/241/1226/376.abstract.

[38] J.D. Eshelby, The Elastic Field Outside an Ellipsoidal Inclusion, Proc. R. Soc. London. Ser. A. Math. Phys. Sci. 252 (1959) 561 LP-569. http://rspa.royalsocietypublishing.org/content/252/1271/561.abstract.

[39] Y.N. Podil'chuk, Deformation of an elastic spheroid, Sov. Appl. Mech. 3 (1967) 24-29. doi:10.1007/BF00886227.

[40] Y.N. Podil'chuk, Stress state of a transversely-isotropic body with elliptical inclusion, Int. Appl. Mech. 33 (1997) 881-887. doi:10.1007/BF02700576.

[41] Y.N. Podil'chuk, Exact analytic solutions of three-dimensional boundary-value problems of the statics of a transversely isotropic body of canonical form (Survey), Int. Appl. Mech. 33 (1997) 763-787. doi:10.1007/BF02719255.

[42] Y.N. Podil'chuk, T.M. Proshchenko, The Stress State of a Piezoceramic Medium with an Elliptic Inclusion under Pure Shear and Pure Bending, Int. Appl. Mech. 39 (2003) 573-582. doi:10.1023/A:1025191609961.

[43] D. Healy, Elastic field in 3D due to a spheroidal inclusion-MATLAB ${ }^{\mathrm{TM}}$ code for Eshelby's solution, Comput. Geosci. 35 (2009) 2170-2173. doi:10.1016/j.cageo.2008.11.012.

[44] L. Boyde, A. Ekpenyong, G. Whyte, J. Guck, Elastic theory for the deformation of a solid or layered spheroid under axisymmetric loading, Acta Mech. 224 (2013) 819-839. doi:10.1007/s00707-0120789-7.

[45] Atlas of Stress-Strain Curves, 2nd Edition, ASM International, Materials Park, Ohio 44073-0002, USA, 2002.

[46] A. Hadjiafxenti, I.E. Gunduz, C. Tsotsos, T. Kyratsi, S.M. Aouadi, C.C. Doumanidis, C. Rebholz, The influence of structure on thermal behavior of reactive Al-Ni powder mixtures formed by ball milling, J. Alloys Compd. 505 (2010) 467-471. doi:10.1016/j.jallcom.2010.03.250.

[47] F. Hausdorff, Dimension und äußeres Maß, Math. Ann. 79 (1919) 157-179. https://eudml.org/doc/158784\#content.

[48] V.M. Reuter, P. Franz, E. Wolter, Fraktaldimension von Grauwertbildern Studienarbeit " $r$ Informatik der Universit a, (1999). 


\section{$\underline{\text { Figure Captions }}$}

Fig. 1: (a) Lamellar structure of ball-milled Ni(bright)-Al(dark) particulate by SEM [5]. (b) Apollonian pack of globular particles (rendered simulation).

Fig. 2: Warped ellipsoid (WE) primitive of monometallic domain.

Fig. 3: Particulate surrounded by particles and clusters and impacted by BM ball (not to scale).

Fig. 4: Model material load-displacement / stress-strain curves. (a) Normal, (b) Shear.

Fig. 5: Laboratory-calibrated Maxwell-Boltzmann pdf of BM ball velocities [23].

Fig. 6: Contact surface pressure and shear distributions. (a) No slip, (b) Partial microslip, (c) Full slip.

Fig. 7: Equivalent stress distribution $\sigma(r)$ during (a) loading and (b) unloading of contact surface.

Fig. 8: Schematic geometry of Al-Ni multi-foil sandwich, and fit in the BM vial (in mm - not to scale).

Fig. 9: Calibration of model surface friction and yield coefficients $(\mu, \eta)$.

Fig. 10: SEM micrograph of Al-Ni bilayer structure after 3 min of ball milling, showing four Al foils (left) bonded to a Ni foil (right). All foils are originally $50 \mu \mathrm{m}$ thick.

Fig. 11: Al-Ni ball-milled particulate structure (Al-dark phase, Ni-light phase): SEM micrographs after (a) 6 h, (b) 12 h, (c) 18 h, (d) 22 h. Model predictions after (e) 6 h, (f) 12 h, (g) 18 h, (h) 22 h. 

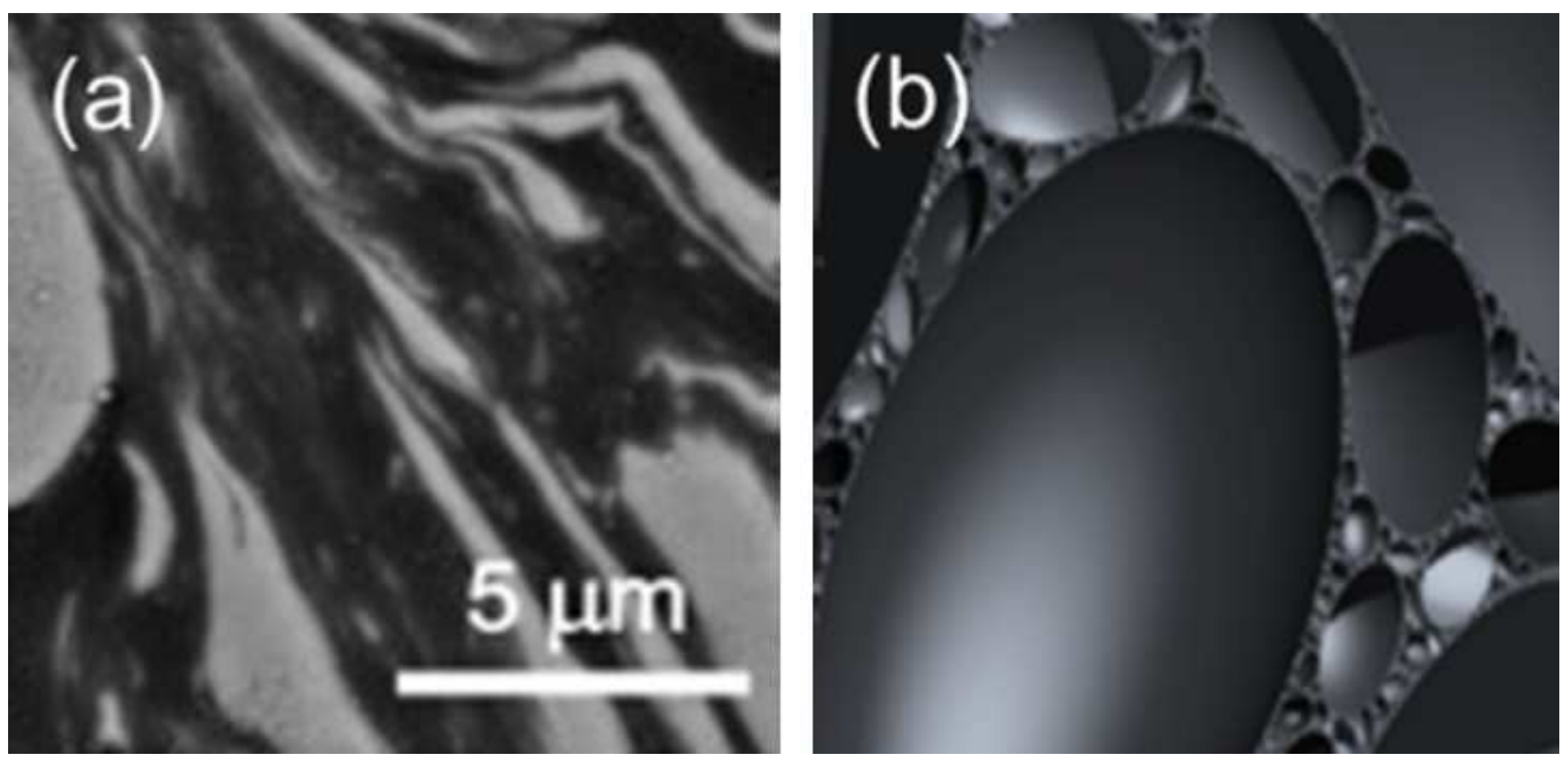
Click here to download high resolution image

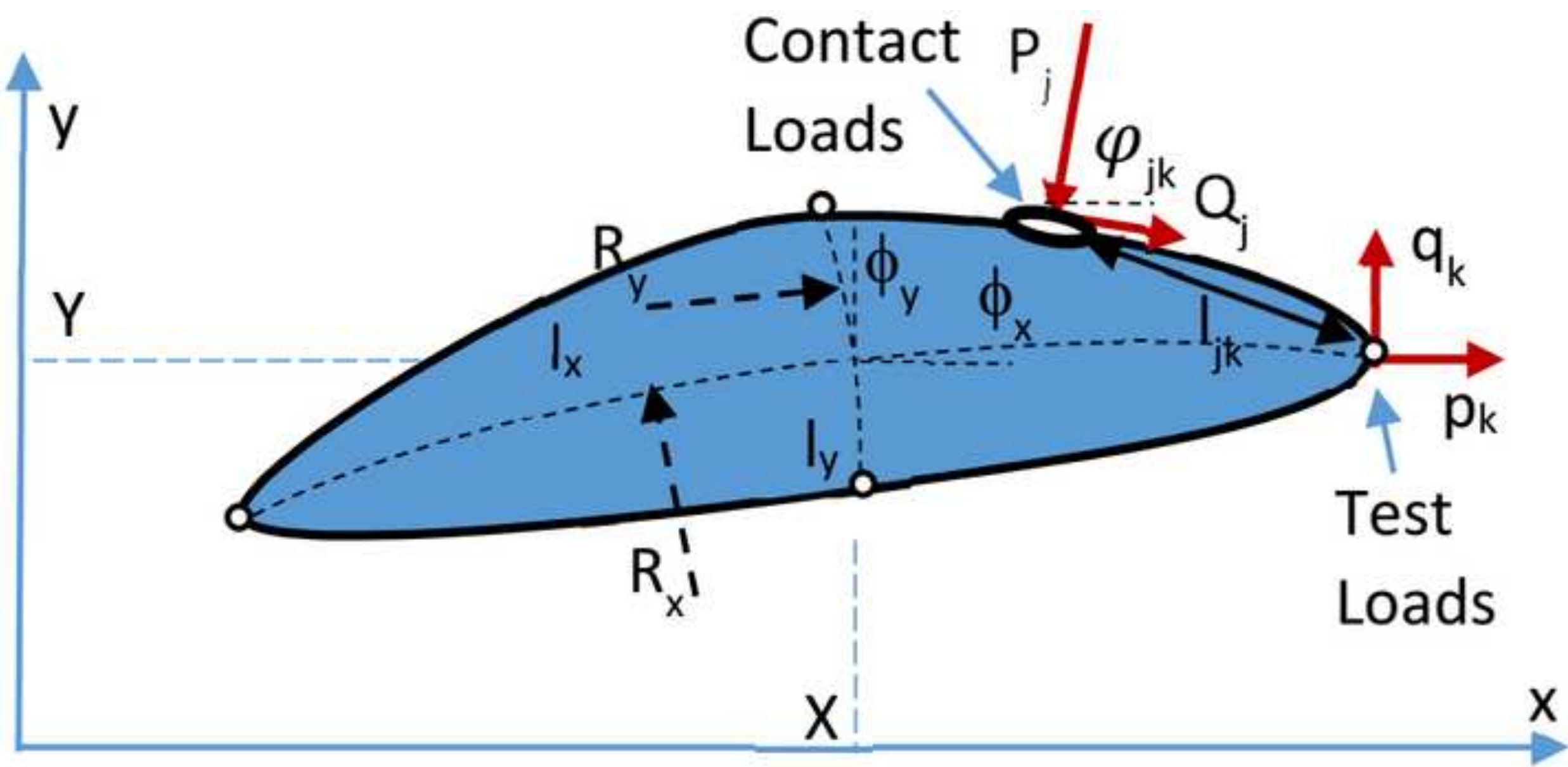

Contact $\mathrm{P}_{\mathrm{j}}$

Loads $\varphi$ 


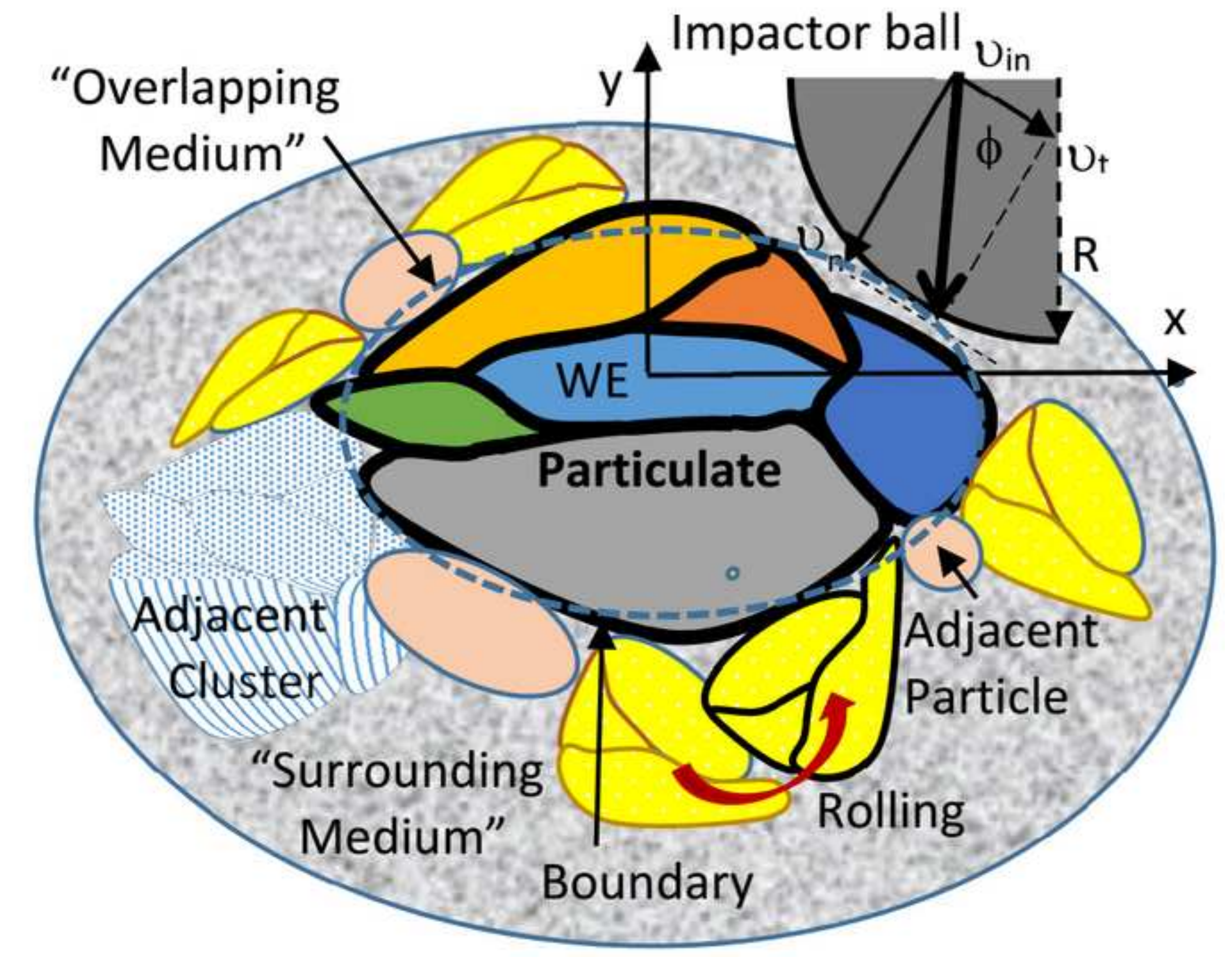


(a) $p \sigma$

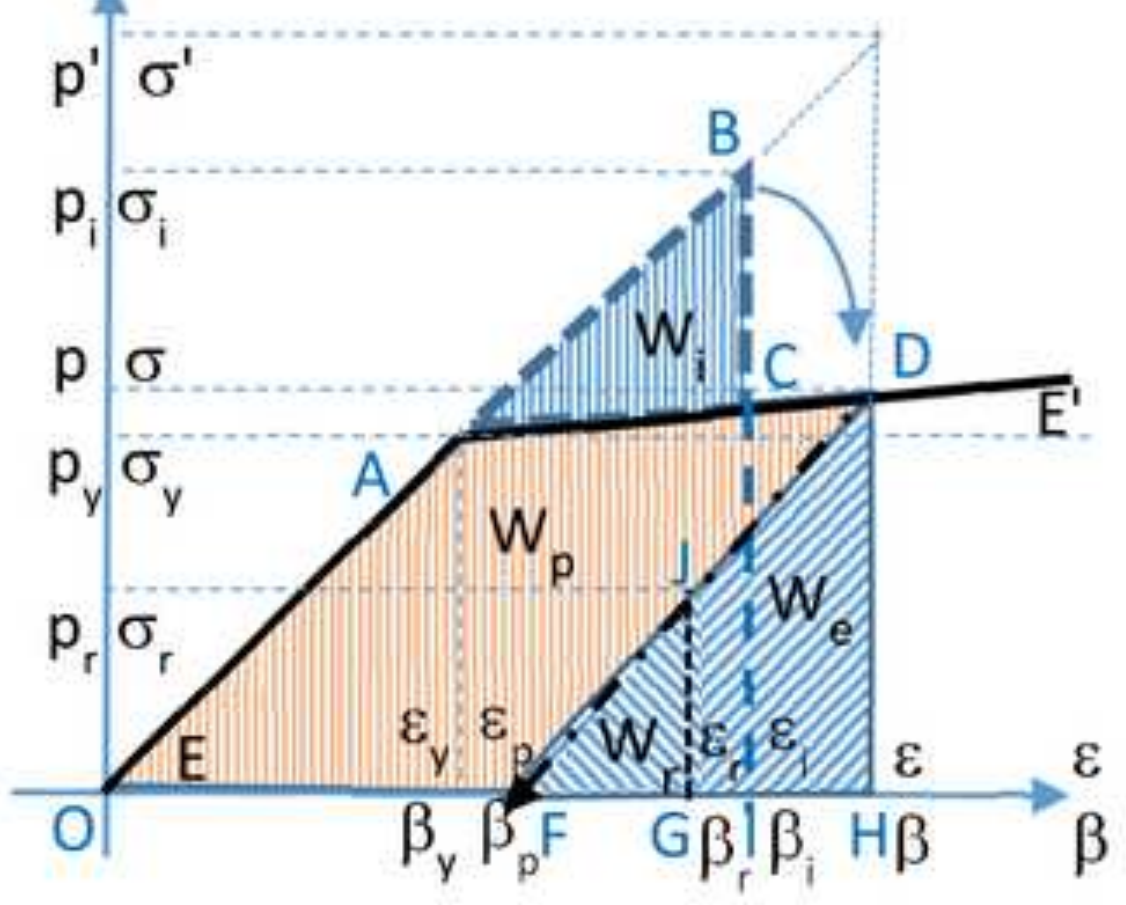

(b) $q \tau$

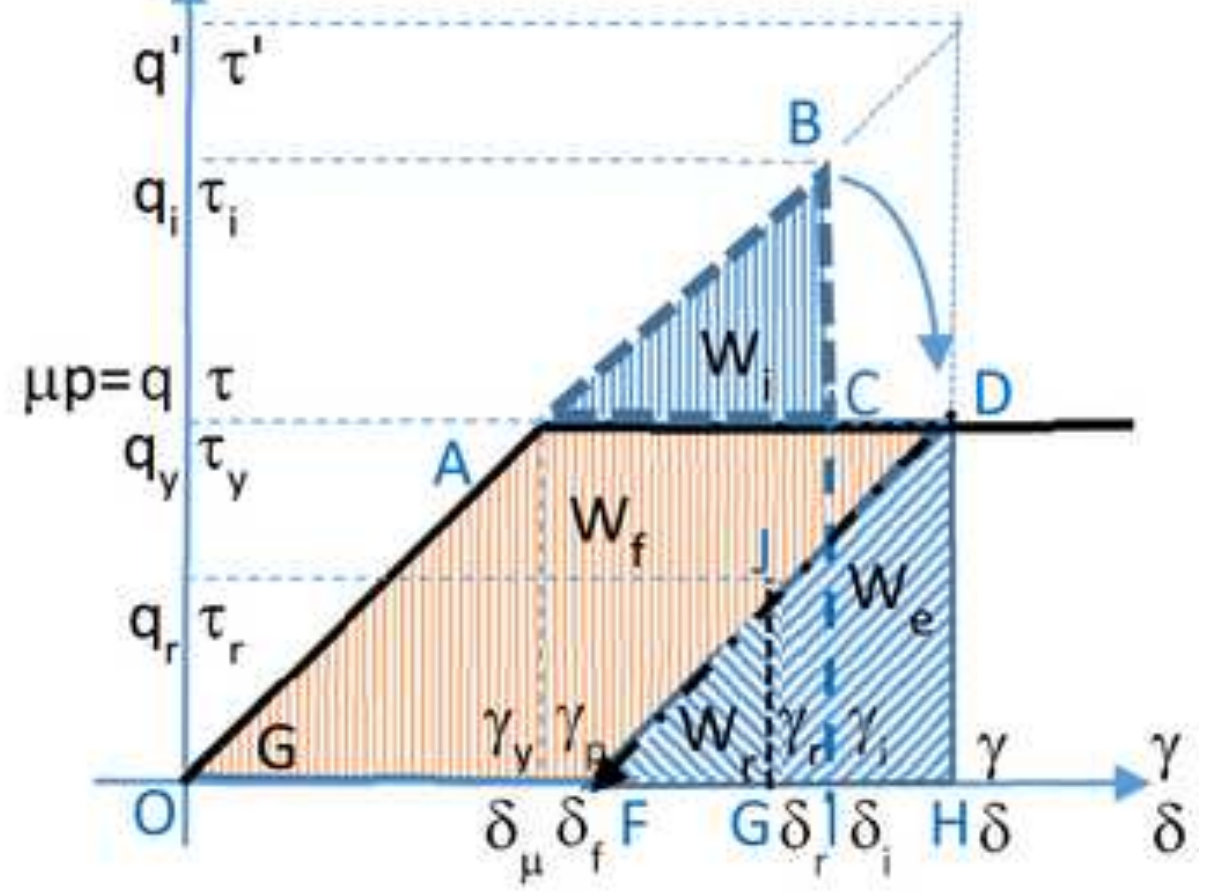




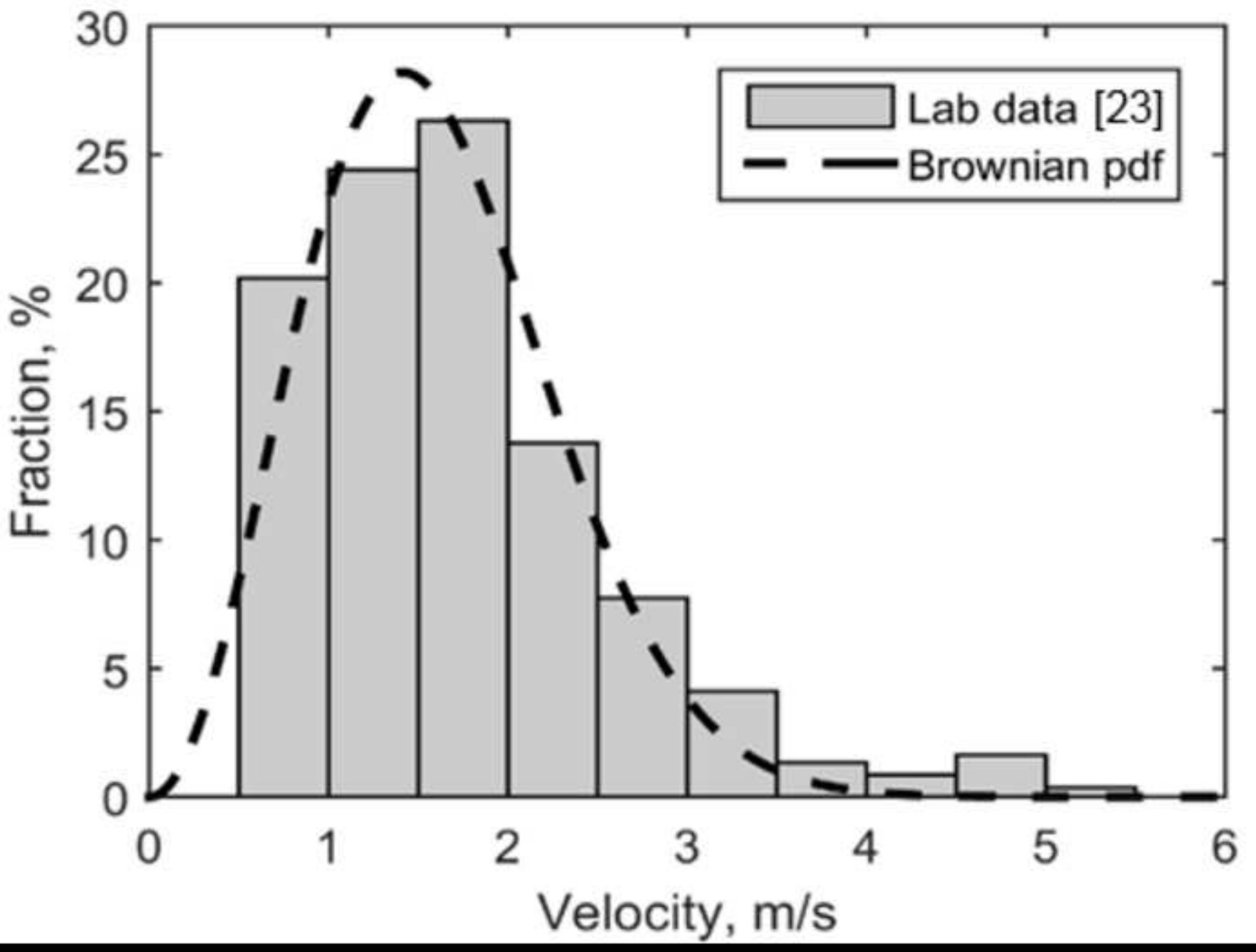



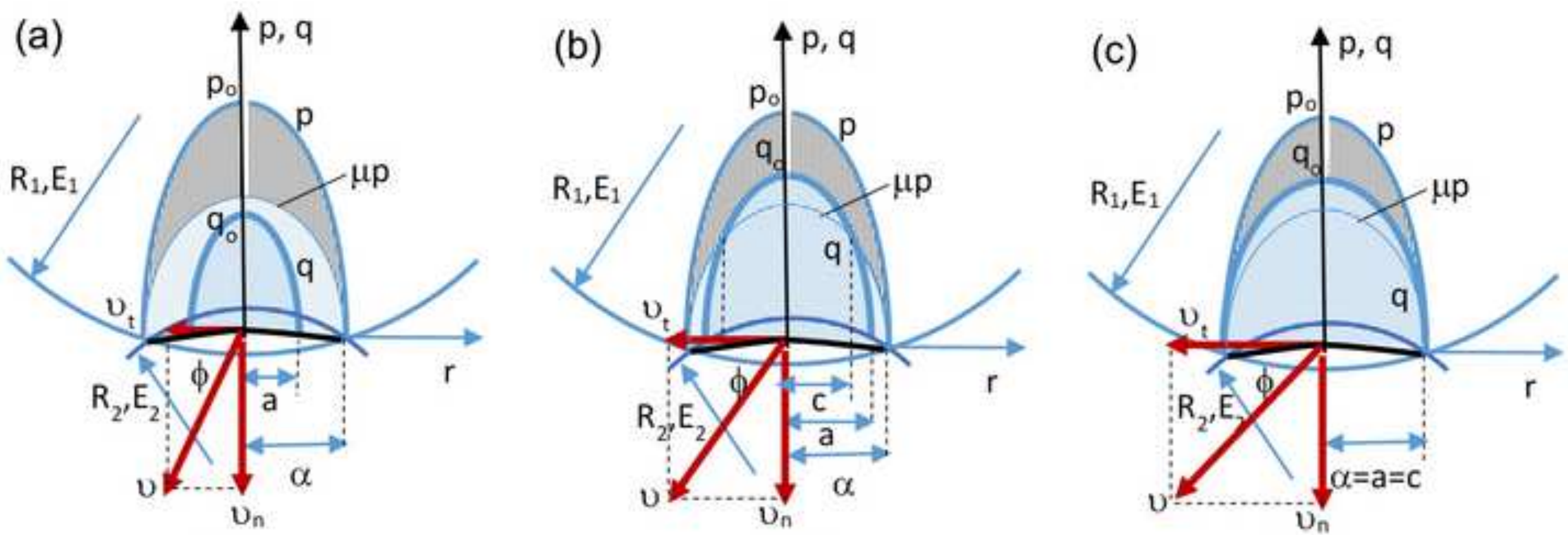
(a)

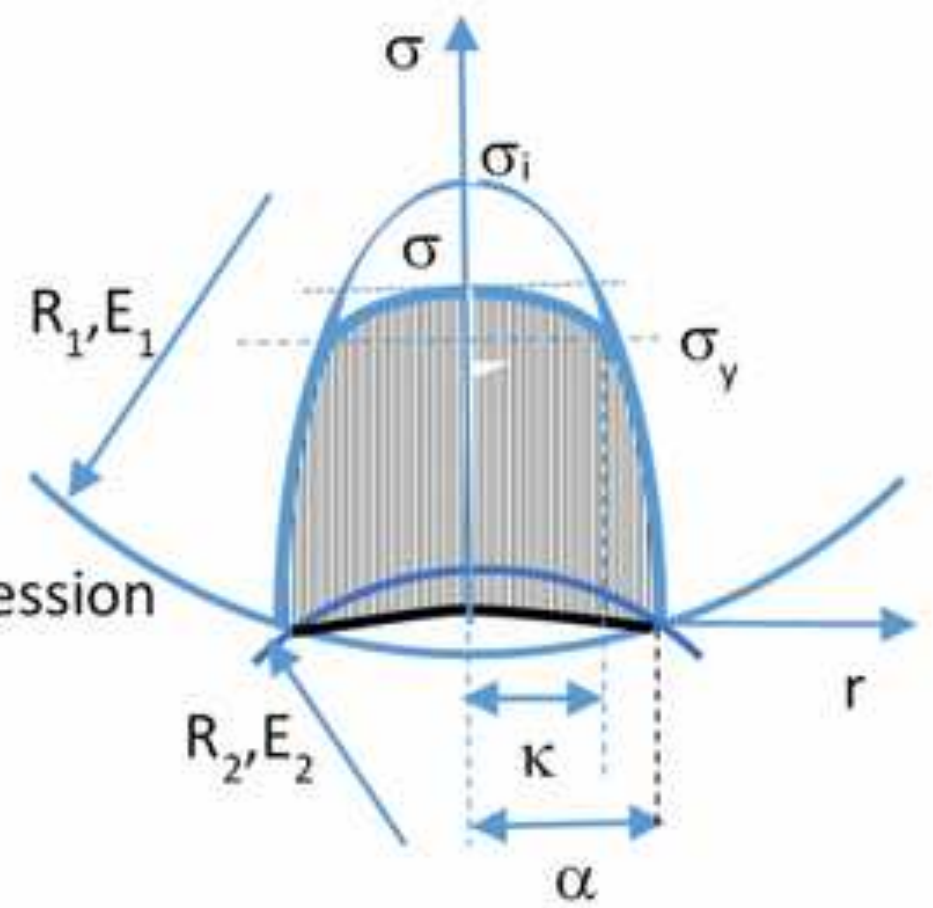

(b)

\section{compression}

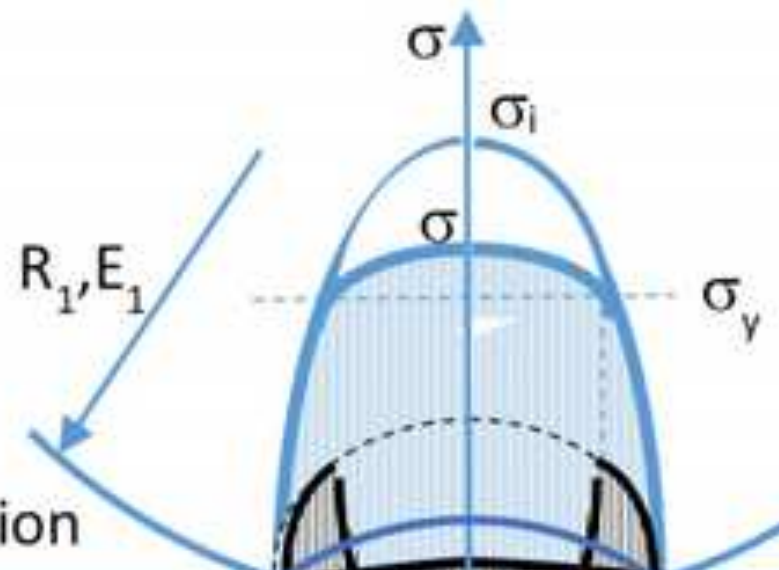

tension 


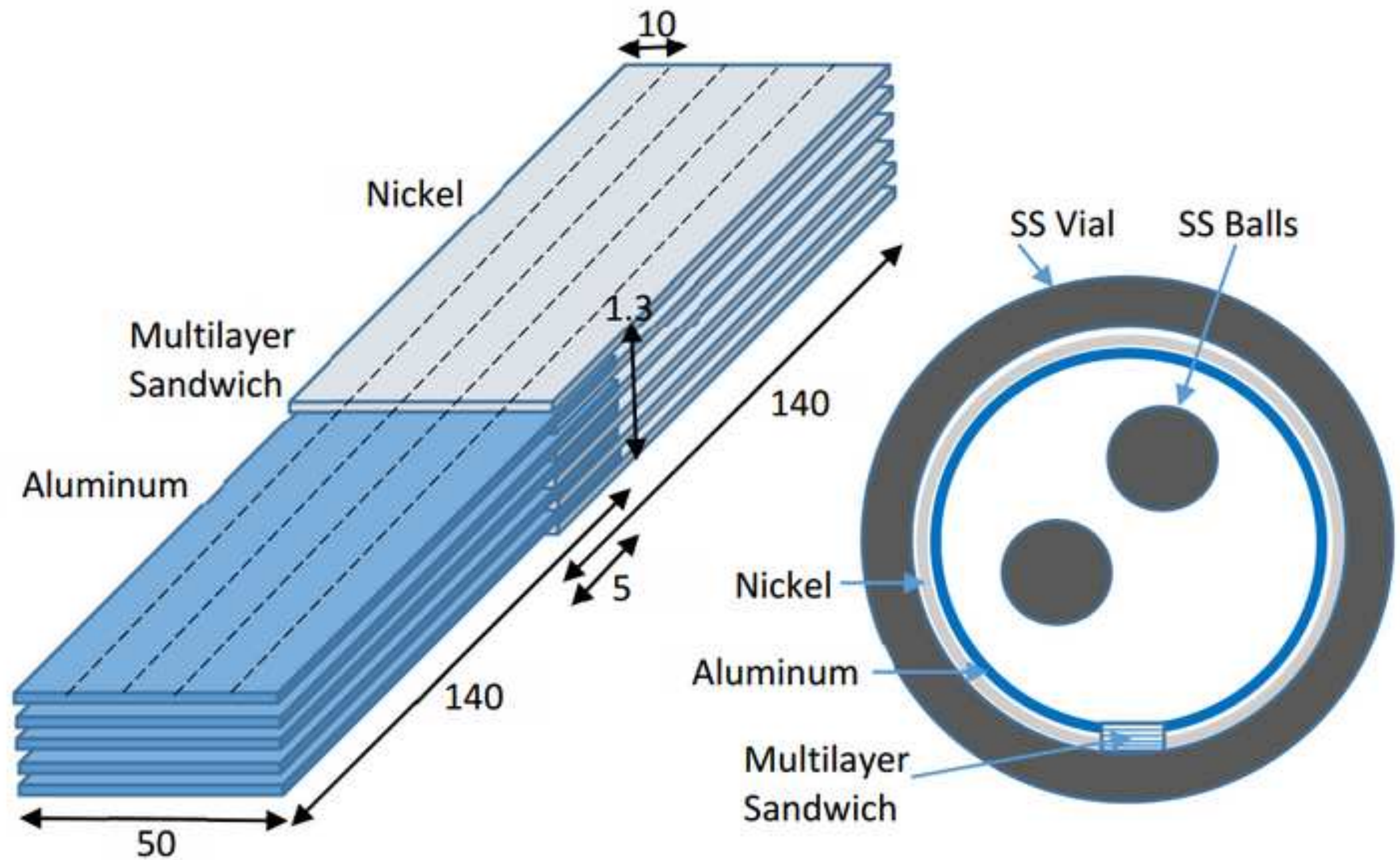




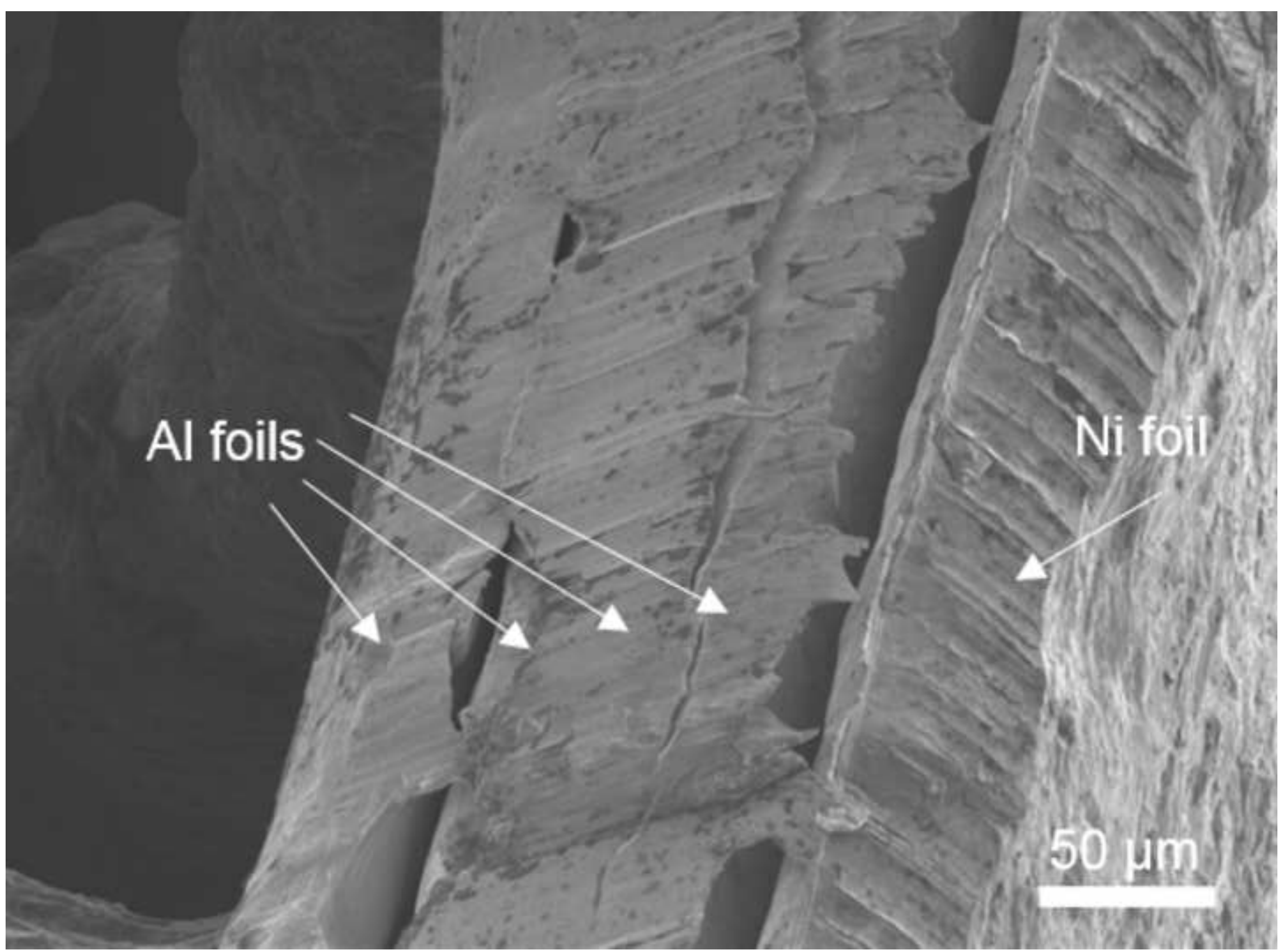

\section{Al foils}


Click here to download high resolution image
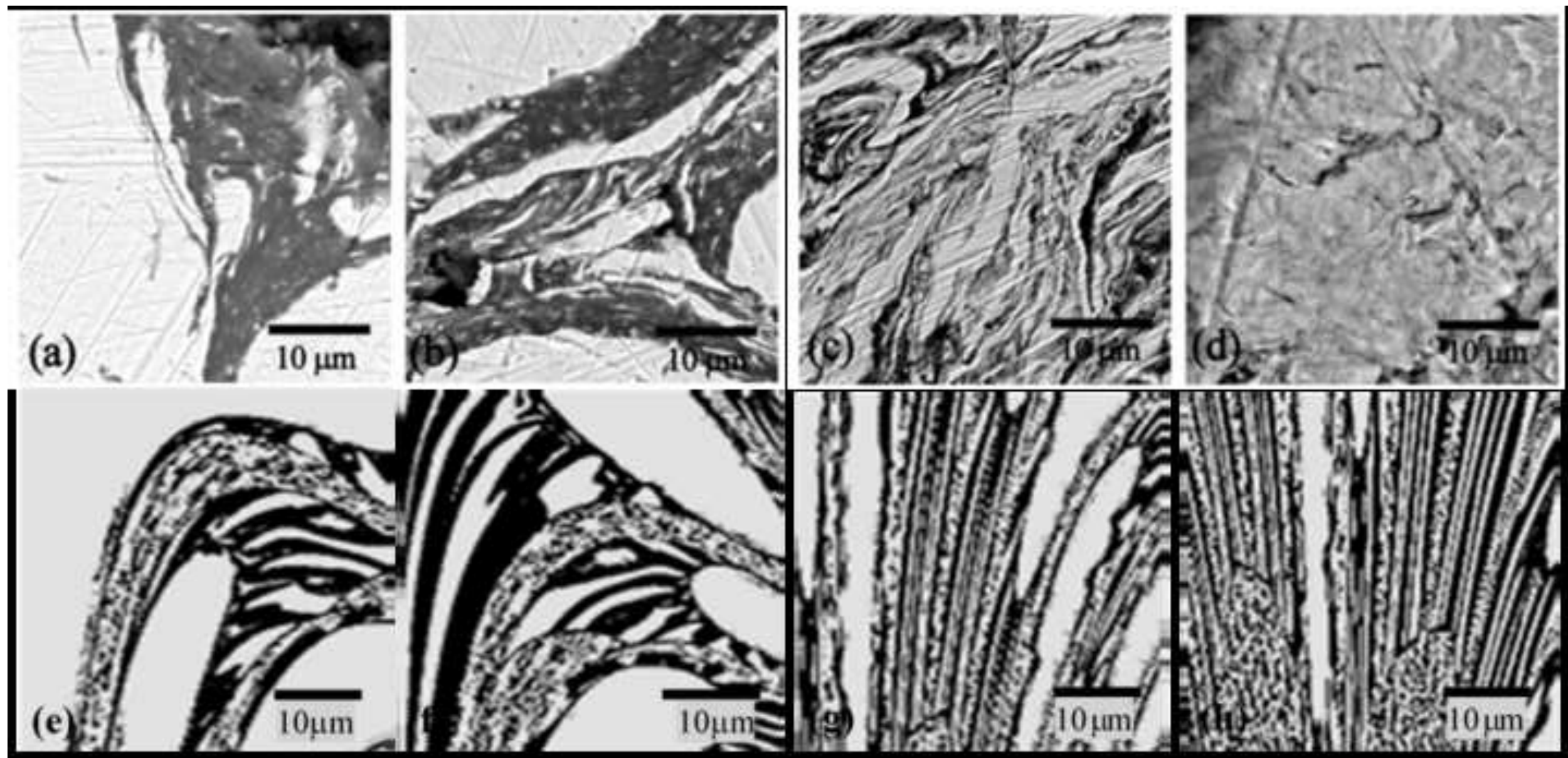
Planetary Ball Milling

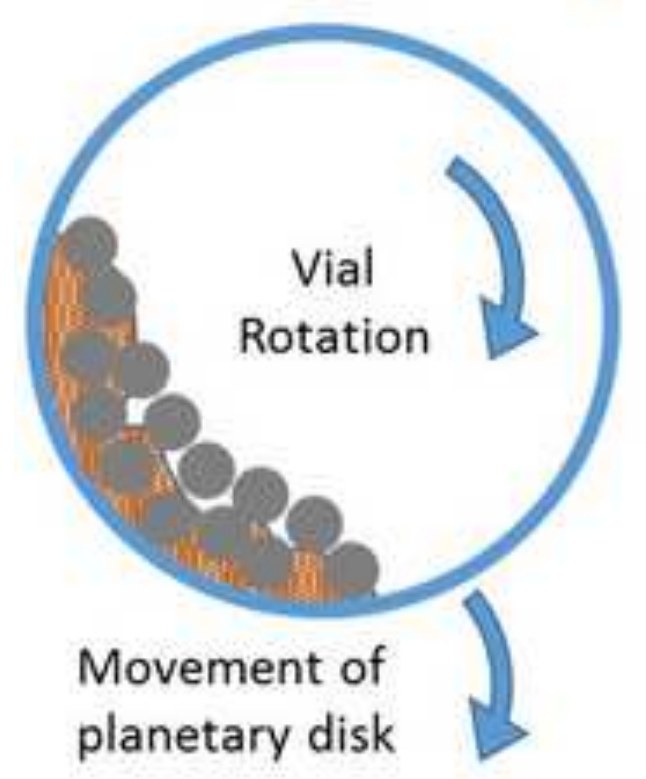

Brownian Ball Motion

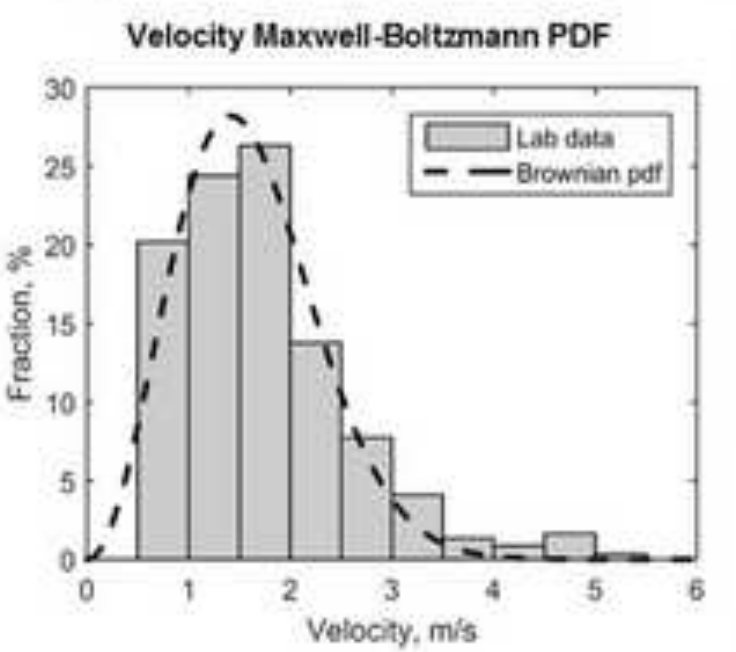

Particle interactions Imaged vs Simulated
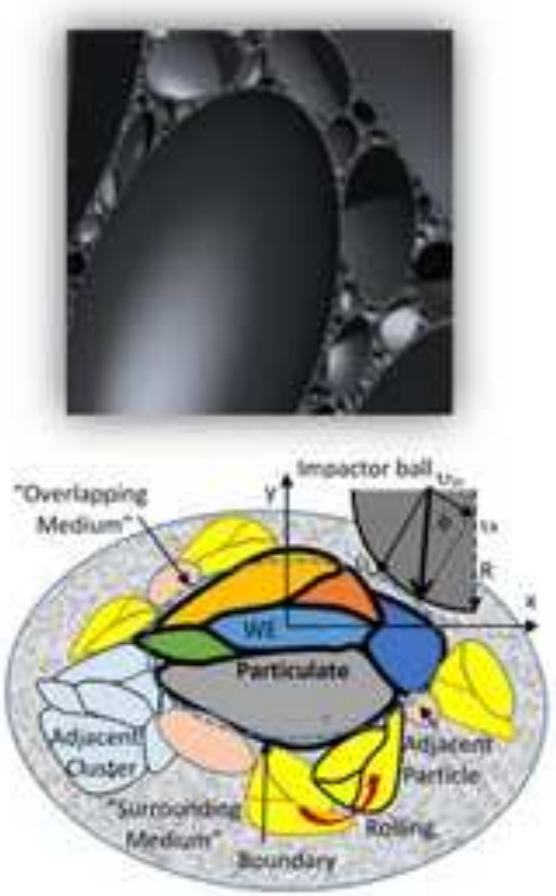

Microstructure
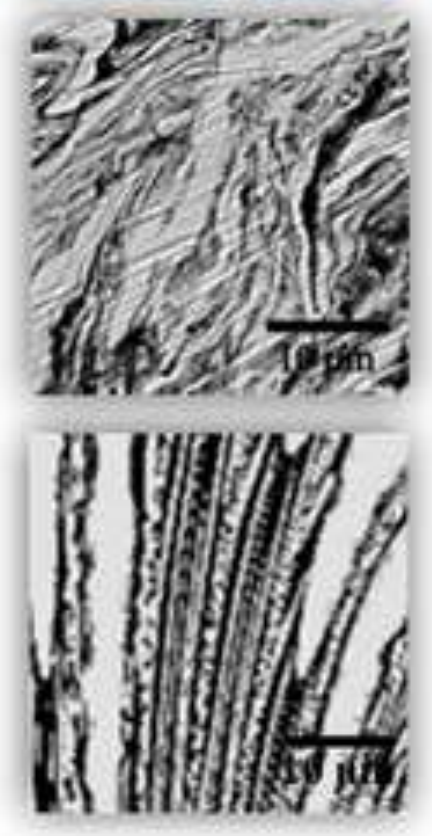